\title{
AN \\ OXATHIAPHOSPHOLANE APPROACH TO ONE-POT PHOSPHOROTHIOYLATION OF ISOPRENOID ALCOHOLS
}

\author{
Katarzyna Zmudzka ${ }^{a}$, Barbara Nawrot ${ }^{a}$, Tadeusz \\ Chojnacki $^{\mathrm{b}}$ and Wojciech J. Stec ${ }^{\mathrm{a} *}$ \\ ${ }^{a}$ Department of Bioorganic Chemistry, Centre of Molecular and \\ Macromolecular Studies, Polish Academy of Sciences, Sienkiewicza 112, \\ 90-363 Lodz, Poland \\ ${ }^{b}$ Institute of Biochemistry and Biophysics, Polish Academy of Sciences, \\ Pawinskiego 5a, 02-106 Warszawa, Poland
}

wjstec@bio.cbmm.lodz.pl

\section{Supporting Information:}

Synthesis of 2-cyanoethoxy-2-thiono-1.3.2-oxathiaphospholane (2). To a solution of 2-cyanoethanol (1 mmol) in pyridine $(20 \mathrm{~mL})$ containing suspended, carefully dried elemental sulfur (2-fold molar excess) was added dropwise, with stirring, an equimolar amount of 2-chloro-1,3,2-oxathiaphospholane. ${ }^{1}$ The reaction progress was monitored by TLC (Merck, silica gel $60 \mathrm{~F}_{254}$ ) using chloroform as eluent. After the reaction was complete $(60 \mathrm{~h}$, room temperature) the pyridine was evaporated under vacuum. Compound 2 was co-evaporated twice with anhydrous toluene and then purified by silica gel column chromatography eluting with chloroform $/ n$ hexane $(1: 1, \mathrm{v} / \mathrm{v})$ followed by chloroform - methanol with increasing gradient of methanol up to $20 \%$. Pure compound 2 was obtained as an pale yellow oil in $73 \%$ yield and its structure was confirmed by HR MS (MW calc. $=208.9741 ; \mathrm{MW}$ exp. $=208.9734),{ }^{31} \mathrm{P} \mathrm{NMR}(\delta 105.6 \mathrm{ppm}),{ }^{1} \mathrm{H}$ NMR $(\delta, \mathrm{ppm}) \mathrm{CDCl}_{3}: 4.39-4.59(\mathrm{~m}, 2 \mathrm{H}$, $\left.-\mathrm{O}-\mathrm{CH}_{2}-\mathrm{CH}_{2}-\mathrm{S}-\right), 4.24-4.33\left(\mathrm{~m}, 2 \mathrm{H},-\mathrm{O}-\mathrm{CH}_{2}-\mathrm{CH}_{2}-\mathrm{CN}\right), 3.48-3.62\left(\mathrm{~m}, 2 \mathrm{H},-\mathrm{O}-\mathrm{CH}_{2}-\underline{\mathrm{CH}}_{2}-\mathrm{S}-\right), 2.75-2.78(\mathrm{t}$, $\left.2 \mathrm{H},{ }^{3} \mathrm{~J}=6.0 \mathrm{~Hz},-\mathrm{O}-\mathrm{CH}_{2}-\mathrm{CH}_{2}-\mathrm{CN}\right)$ and ${ }^{13} \mathrm{C} \mathrm{NMR}(\delta, \mathrm{ppm}) \mathrm{CDCl}_{3}: 116.5\left(-\mathrm{O}-\mathrm{CH}_{2}-\mathrm{CH}_{2}-\underline{\mathrm{CN}}\right), 70.9\left(-\mathrm{O}-\mathrm{CH}_{2}-\mathrm{CH}_{2}-\right.$ $\mathrm{S}-), 62.29\left(\mathrm{~d},{ }^{2} \mathrm{~J}_{\mathrm{P}-\mathrm{C}}=5.75 \mathrm{~Hz},-\mathrm{O}-\underline{\mathrm{CH}}_{2}-\mathrm{CH}_{2}-\mathrm{CN}\right), 36.39\left(-\mathrm{O}-\mathrm{CH}_{2}-\underline{\mathrm{CH}}_{2}-\mathrm{S}-\right), 19.42\left(\mathrm{~d},{ }^{3} \mathrm{~J}_{\mathrm{P}-\mathrm{C}}=8.20 \mathrm{~Hz},-\mathrm{O}-\mathrm{CH}_{2}-\right.$ $\underline{\mathrm{CH}}_{2}-\mathrm{CN}$ ). TLC (silica gel $\mathrm{HF}_{254}$, Merck) in $\mathrm{CHCl}_{3}, \mathrm{v} / \mathrm{v}: \mathrm{R}_{\mathrm{f}}=0.53$.

One-pot phosphorothioylation of citronellyl alcohol 1d: To a solution of compound 2 (209 mg, $1 \mathrm{mmol}) \mathrm{in}$ anhydrous acetonitrile $(2 \mathrm{~mL})$ was added an equimolar amount of compound $\mathbf{1 d}(182 \mu \mathrm{L}, 1 \mathrm{mmol})$. The resulting mixture was cooled to $0{ }^{\circ} \mathrm{C}$ with an external bath and a stoichiometric amount of DBU (149 $\left.\mu \mathrm{L}, 1 \mathrm{mmol}\right)$ was added dropwise, with stirring. The cooling bath was removed and reaction mixture was left for $30 \mathrm{~min}$ at ambient temperature. The acetonitrile was evaporated under reduced pressure and the resulting 4d was treated with $28 \%$ aqueous ammonia / dioxane $1: 1(\mathrm{v} / \mathrm{v})$ mixture $(2 \mathrm{~mL})$ for $48 \mathrm{~h}$ at room temperature. Crude 5d was placed on a silica gel column and eluted with propan-2-ol / water / $28 \%$ aq. ammonia (7:1:1). TLC analysis

\footnotetext{
${ }^{1}$ Martynov, I.V.; Kruglyak, Yu.L.; Leibovskaya, G.A.,; Khromova, Z.I.; Stukov, O.G.; Zh. Obshch. Khim., 1969, $39,966$.
} 
(silica gel $\mathrm{HF}_{254}$, Merck) of purified product was performed in propan-2-ol / water / $28 \%$ aq. ammonia (7:1:1) and staining was done with $0.5 \%$ palladium chloride solution in water containing $0.05 \%$ conc. $\mathrm{HCl}$. $156 \mathrm{mg}$ of 5d was obtained as an colorless oil (yield $62 \%$ ).

The remaining alcohols were phosphorothioylated as described above. The yields of the transformation of 1a-f to $\mathbf{5 a - f}$ and spectral characteristics of $\mathbf{4 a - f}\left({ }^{31} \mathrm{P}\right.$ NMR in a crude reaction mixture) and $\mathbf{5 a - f}\left({ }^{31} \mathrm{P}\right.$ NMR and HR MS) are given in Table 1.

TLC $\mathbf{R}_{\mathbf{f}}$ of 5a-5f in propan-2-ol / water / $28 \%$ aq. ammonia (7:1:1) are as follow: 5a: 0.27; 5b: 0.39; 5c: 0.30; 5d: 0.20 ; 5e: $0.15 ; \mathbf{5 f :} 0.30$.

${ }^{1} \mathbf{H}$ NMR $\left(\delta\right.$, ppm, $\left.\mathrm{D}_{2} \mathrm{O}\right)$ data for phosphorothioate moieties of $\mathbf{5}$ are as follow:

5a: 6.01 (ddt, $1 \mathrm{H},{ }^{3} \mathrm{~J}_{\mathrm{HcHa}}=17.2 \mathrm{~Hz},{ }^{3} \mathrm{~J}_{\mathrm{HcHb}}=10.3 \mathrm{~Hz}$ and $\left.{ }^{3} \mathrm{~J}_{\mathrm{HcHd}, \mathrm{e}}=5.3 \mathrm{~Hz}, \mathrm{CH}_{2}=\mathrm{C}_{\mathrm{H}_{\mathrm{c}}}-\mathrm{CH}_{2}-\mathrm{O}-\right), 5.36$ (ddt, ${ }^{3} \mathrm{~J}_{\mathrm{HcHa}}=17.2$ $\mathrm{Hz},{ }^{3} \mathrm{~J}_{\mathrm{HbHa}}=3.0 \mathrm{~Hz}$ and $\left.{ }^{3} \mathrm{~J}_{\mathrm{HaHd}, \mathrm{e}}=1.7 \mathrm{~Hz}, 1 \mathrm{H}, \underline{\mathrm{H}}_{2} \mathrm{CH}=\mathrm{CH}-\mathrm{CH}_{2}-\mathrm{O}-\right), 5.20\left(\mathrm{ddt},{ }^{3} \mathrm{~J}_{\mathrm{HbHc}}=10.3 \mathrm{~Hz},{ }^{3} \mathrm{~J}_{\mathrm{HbHa}}=3.0 \mathrm{~Hz}\right.$ and $\left.{ }^{3} \mathrm{~J}_{\mathrm{HbHd}, \mathrm{e}}=1.4 \mathrm{~Hz}, 1 \mathrm{H}, \mathrm{HC}_{\mathrm{b}}=\mathrm{CH}-\mathrm{CH}_{2}-\mathrm{O}-\right), 4.36\left(\mathrm{~m}, \mathrm{CH}_{2}=\mathrm{CH}-\underline{\mathrm{H}}_{2(\mathrm{~d}, \mathrm{e})}-\mathrm{O}-\right)$;

5b: 4.82-4.90 (m, 2H, $\left.\underline{\mathrm{CH}}_{2}=\mathrm{C}-\right), 3.96\left(\mathrm{dt},{ }^{3} \mathrm{~J}_{\mathrm{H}-\mathrm{P}}=7.1 \mathrm{~Hz},{ }^{3} \mathrm{~J}_{\mathrm{H}-\mathrm{H}}=7.0 \mathrm{~Hz}, 2 \mathrm{H},-\mathrm{CH}_{2}-\mathrm{CH}_{2}-\mathrm{O}-\right), 2.37\left(\mathrm{t}, 2 \mathrm{H},{ }^{3} \mathrm{~J}=7.0\right.$ $\left.\mathrm{Hz}, \underline{\mathrm{C}}_{2}-\mathrm{CH}_{2}-\mathrm{O}-\right), 1.77$ (s, 3H, $\left.\underline{\mathrm{C}}_{3}\right)$;

$\mathbf{5 c}^{2}: 5.44\left(\mathrm{t}, 1 \mathrm{H},{ }^{3} \mathrm{~J}=7.0 \mathrm{~Hz}, \mathrm{C}=\mathrm{C} \underline{H}-\mathrm{CH}_{2}-\right), 5.22-5.25\left(\mathrm{~m}, 1 \mathrm{H},\left(\mathrm{CH}_{3}\right)-\mathrm{C}=\mathrm{C} \underline{H}-\mathrm{CH}_{2}-\right), 4.42\left(\mathrm{dd}, 2 \mathrm{H},{ }^{3} \mathrm{~J}_{\mathrm{H}-\mathrm{P}}=7.0 \mathrm{~Hz}\right.$, $\left.{ }^{3} \mathrm{~J}_{\mathrm{H}-\mathrm{H}}=6.9 \mathrm{~Hz},-\underline{\mathrm{C}}_{2}-\mathrm{O}-\right), 1.98-2.15\left(\mathrm{~m}, 4 \mathrm{H},-\mathrm{CH}-\underline{\mathrm{H}}_{2}-\underline{\mathrm{C}}_{2}-\mathrm{C}\right), 1.72\left(\mathrm{~s}, 3 \mathrm{H}, \underline{\mathrm{C}}_{3}\right), 1.70\left(\mathrm{~s}, 3 \mathrm{H}, \underline{\mathrm{C}}_{3}\right), 1.64(\mathrm{~s}, 3 \mathrm{H}$, $\left.\mathrm{CH}_{3}\right)$;

5d: 5.23-5.24 (br.t, $\left.1 \mathrm{H}, \mathrm{C}=\mathrm{C} \underline{\mathrm{H}}-\mathrm{CH}_{2}-\right), 3.85-3.95\left(\mathrm{dt},{ }^{3} \mathrm{~J}_{\mathrm{H}-\mathrm{P}}=7.1 \mathrm{~Hz},{ }^{3} \mathrm{~J}_{\mathrm{H}-\mathrm{H}}=5.2 \mathrm{~Hz}, 2 \mathrm{H},-\mathrm{CH}_{2}-\mathrm{C}_{2}-\mathrm{O}-\right), 1.96-2.07$ (m, $\left.2 \mathrm{H},-\mathrm{CH}_{2}-\mathrm{CH}_{2}-\mathrm{O}-\right), 1.68$ (s, 3H, $\left.\underline{\mathrm{CH}}_{3}\right), 1.67$ (s, 3H, $\left.\underline{\mathrm{C}}_{3}\right), 1.71$ (m, 1H, - $\left.\mathrm{CH}_{2}-\mathrm{CH}-\left(\mathrm{CH}_{3}\right)-\right), 1.13-1.50$ (m, 4H, $\left.-\mathrm{CH}-\mathrm{CH}_{2}-\underline{\mathrm{CH}}_{2}-\mathrm{CH}-\right), 1.00\left(\mathrm{~d},{ }^{3} \mathrm{~J}_{\mathrm{H}-\mathrm{H}}=5.5 \mathrm{~Hz}, 3 \mathrm{H},-\mathrm{CH}_{2}-\mathrm{CH}-\left(\mathrm{CH}_{3}\right)-\mathrm{CH}_{2}-\right)$;

5e: 5.55 (br. $\left.\mathrm{s}, 1 \mathrm{H},-\mathrm{CH}_{2}-\left(\mathrm{CH}_{3}\right)-\mathrm{C}=\mathrm{C} \underline{\mathrm{H}}-\mathrm{CH}_{2}-\mathrm{O}-\right), 5.22-5.32\left(\mathrm{~m}, 2 \mathrm{H},\left(\mathrm{CH}_{3}\right)_{2}-\mathrm{C}=\mathrm{C} \underline{\mathrm{H}}-\mathrm{CH}_{2} \mathrm{CH}_{2}-\mathrm{C}\left(\mathrm{CH}_{3}\right)=\mathrm{C} \underline{\mathrm{H}}\right), 4.40$

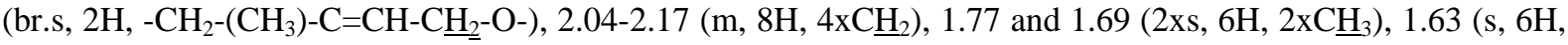
$\left.2 \mathrm{xC} \underline{\mathrm{H}}_{3}\right)$;

5f: $\left(\mathrm{D}_{2} \mathrm{O} / \mathrm{Py}-d_{6}, 1: 1\right): 5.26$ (br. $\left.\mathrm{s}, 1 \mathrm{H},-\mathrm{CH}_{2}-\left(\mathrm{CH}_{3}\right)-\mathrm{C}=\mathrm{C} \underline{\mathrm{H}}-\mathrm{CH}_{2}-\mathrm{O}-\right), 4.34$ (br. d, ${ }^{3} \mathrm{~J}_{\mathrm{H}-\mathrm{P}}=5.7 \mathrm{~Hz}, 2 \mathrm{H},-\mathrm{CH}_{2}-\left(\mathrm{CH}_{3}\right)-$ $\mathrm{C}=\mathrm{CH}-\underline{\mathrm{H}}_{2}-\mathrm{O}-$ ), 1.65 (br. s, $1 \mathrm{H},-\mathrm{C} \underline{\mathrm{H}}-$ ), 1.64 (br. s, $1 \mathrm{H},-\mathrm{C} \underline{\mathrm{H}}-$ ), 1.42 (br. s, $3 \mathrm{H}, \mathrm{CH}_{3}$ ), 1.32 (br. s, 1H, - $\mathrm{C}-$-), 0.78-1.07 (m, 18H, 3x - $\underline{\mathrm{H}}_{2}-\underline{\mathrm{C}}_{2}-\underline{\mathrm{C}}_{2}-$ ), 0.67 and 0.66 ( $2 \mathrm{x}$ br. s, $9 \mathrm{H}, 3 \mathrm{XCH}_{3}$ ), 0.57 (br. s, $3 \mathrm{H}, \mathrm{CH}_{3}$ );

In spectra of $\mathbf{5 a}, \mathbf{5 b}, \mathbf{5 c}, \mathbf{5 d}$ and $\mathbf{5 f}$ the following signals corresponding to DBU-D ${ }^{+}$cation can be found: $3.54\left(\mathrm{q}, 4 \mathrm{H},{ }^{3} \mathrm{~J}=6 \mathrm{~Hz}\right), 3.32\left(\mathrm{t}, 2 \mathrm{H},{ }^{3} \mathrm{~J}=6 \mathrm{~Hz}\right), 2.61-2.65(\mathrm{~m}, 2 \mathrm{H}), 1.98-2.04(\mathrm{~m}, 2 \mathrm{H}), 1.64-1.72(\mathrm{~m}, 6 \mathrm{H})$;

$\mathrm{NH}_{4}{ }^{+}$cation can be found in spectra of $\mathbf{5}$ taken in DMSO- $d_{6}$ (for $\mathbf{5 a} \delta=7.69 \mathrm{ppm}$ ).

\footnotetext{
${ }^{2}$ Mautz, D.S.; Davidson, V.J.; Poulter, C.D. Tetrahedron Lett. 1989, 30, 7333.
} 


\section{Spectral data of compound 2.}

Compound 2: Selected regions of $2 \mathrm{D}^{1} \mathrm{H}-{ }^{1} \mathrm{H}$ COSY spectrum

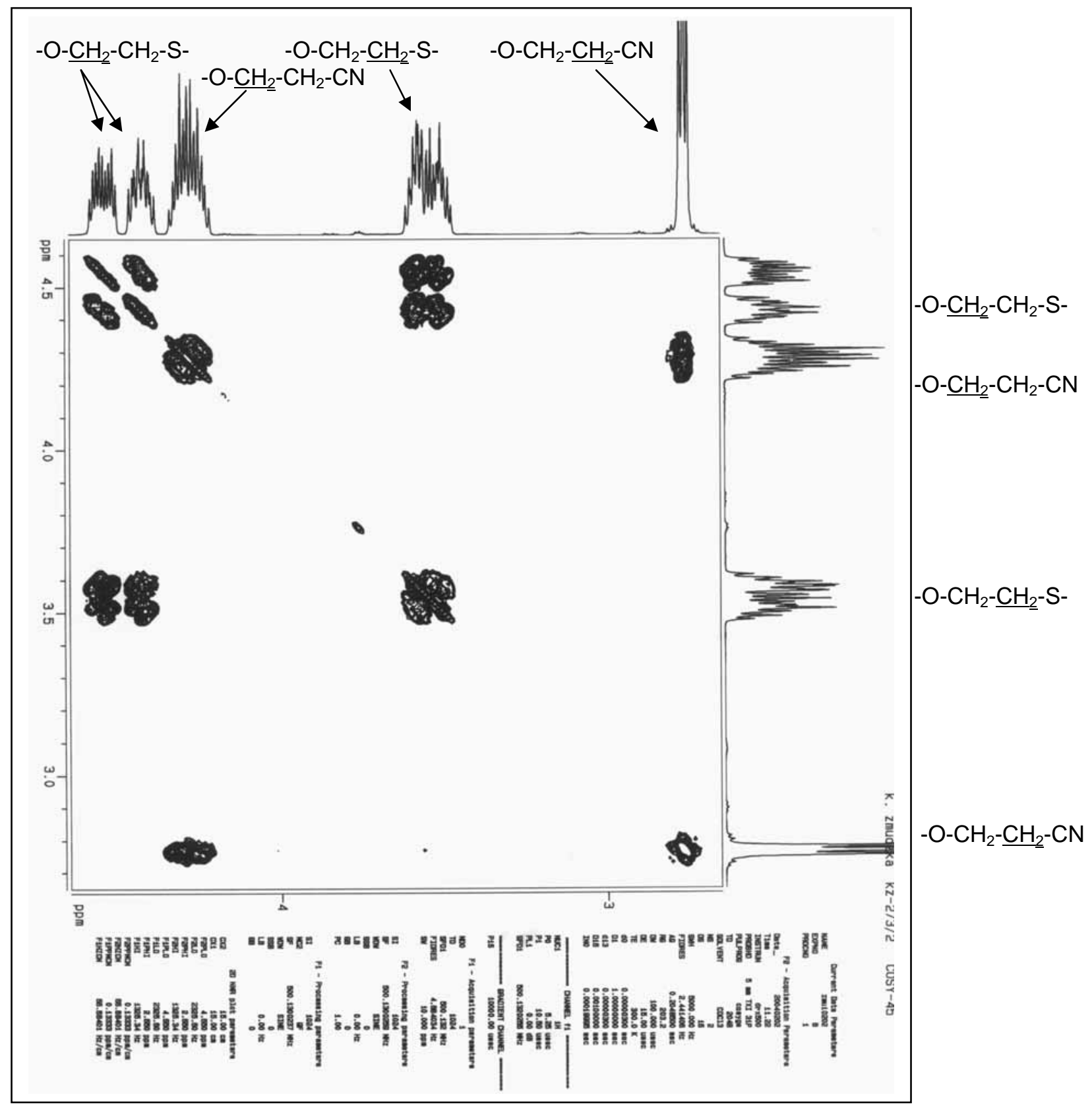


Compound 2: Selected regions of $2 \mathrm{D}{ }^{1} \mathrm{H}-{ }^{13} \mathrm{C}$ HSQC spectrum

$-\mathrm{O}-\mathrm{CH}_{2}-\mathrm{CH}_{2}-\mathrm{S}-\quad-\mathrm{O}-\mathrm{CH}_{2}-\mathrm{CH}_{2}-\mathrm{CN} \quad-\mathrm{O}-\mathrm{CH}_{2}-\mathrm{CH}_{2}-\mathrm{S}-\quad-\mathrm{O}-\mathrm{CH}_{2}-\mathrm{CH}_{2}-\mathrm{CN}$

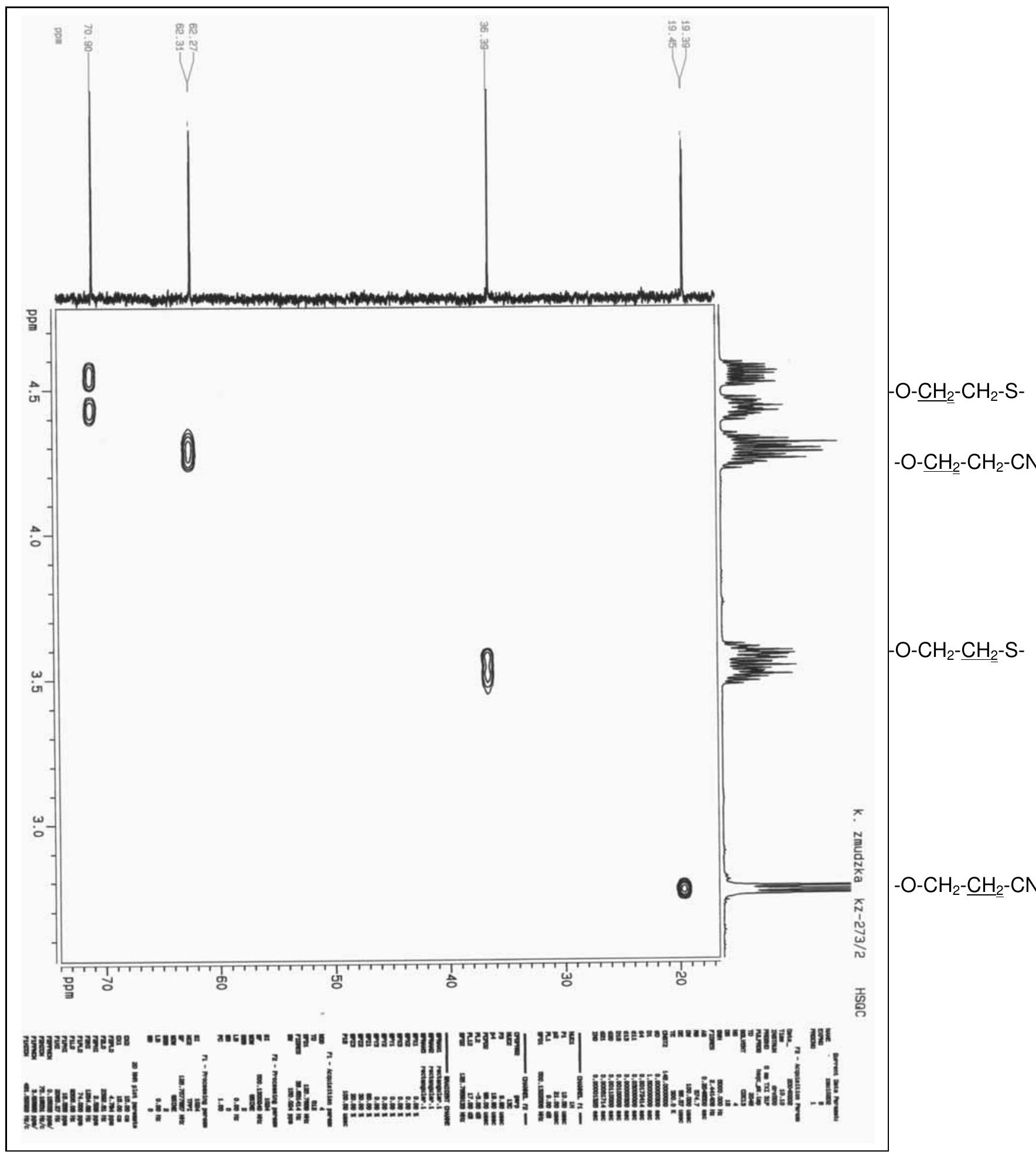


Compound 2: ${ }^{1} \mathrm{H}$ NMR spectrum

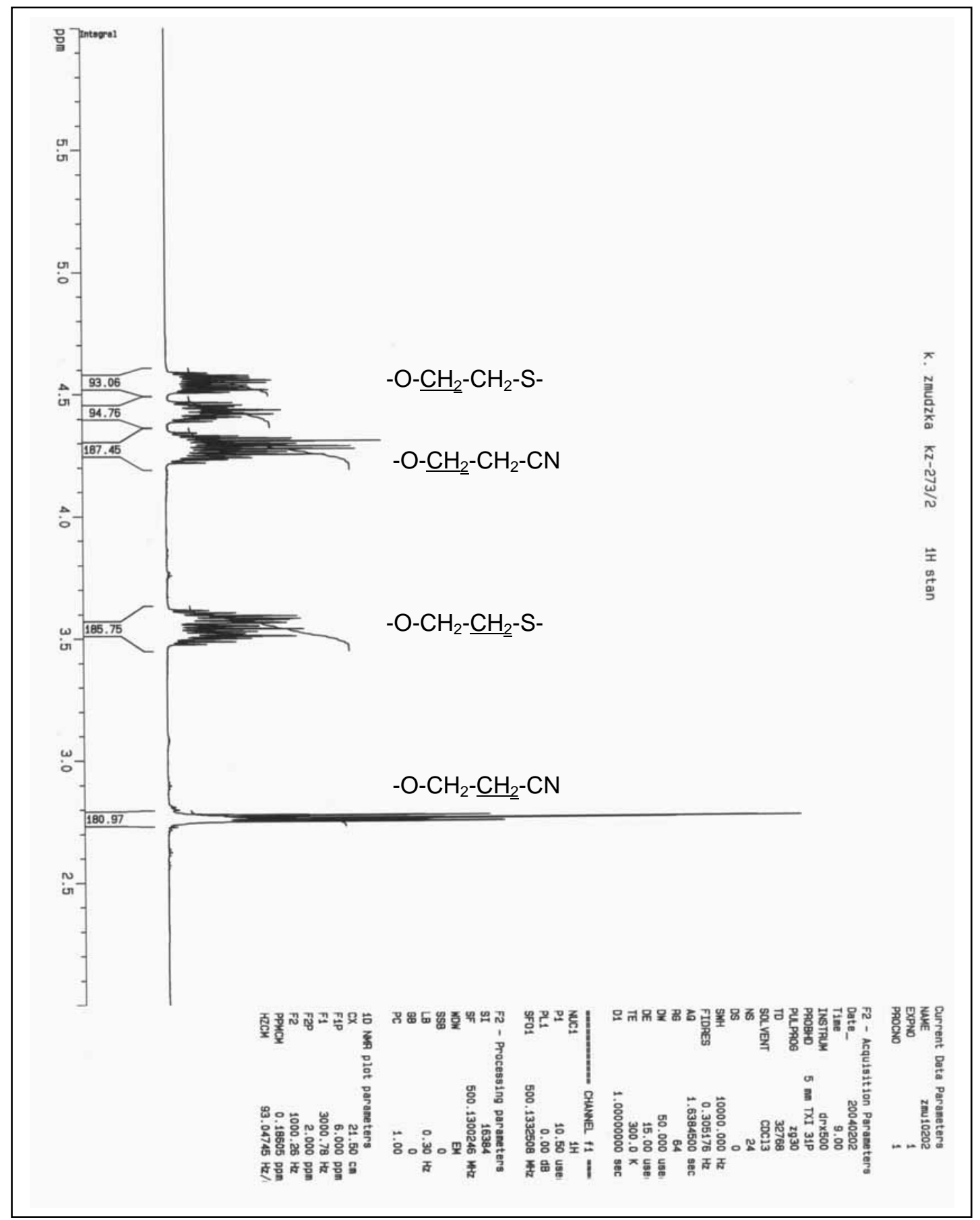


Compound 2: ${ }^{1} \mathrm{H}$ NMR spectrum with phosphorus decoupling

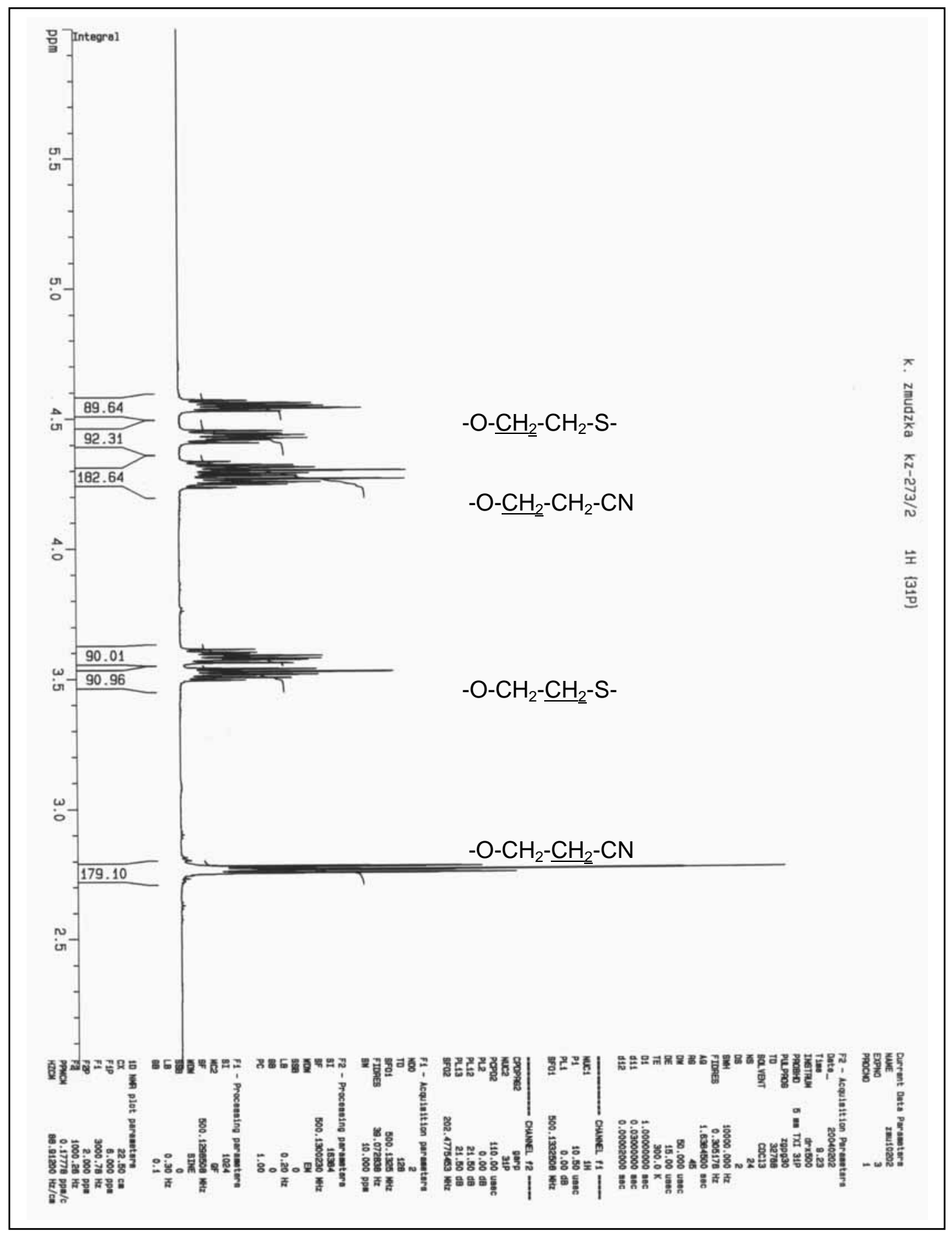


Compound 2: Detailed comparison of ${ }^{1} \mathrm{H}$ NMR spectra without and with ${ }^{31} \mathrm{P}$ decoupling

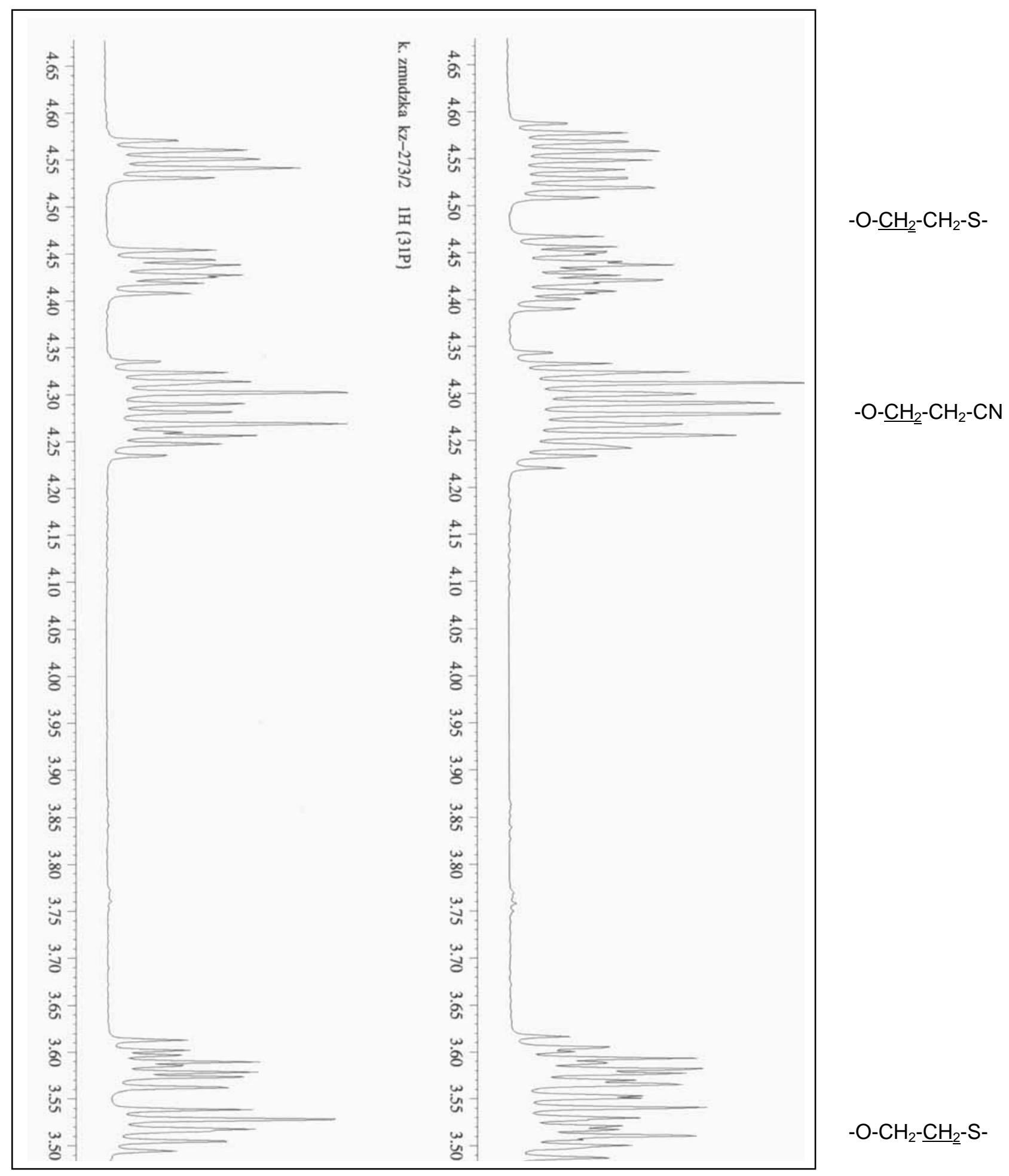


Compound 2: ${ }^{13} \mathrm{C}$ NMR spectrum

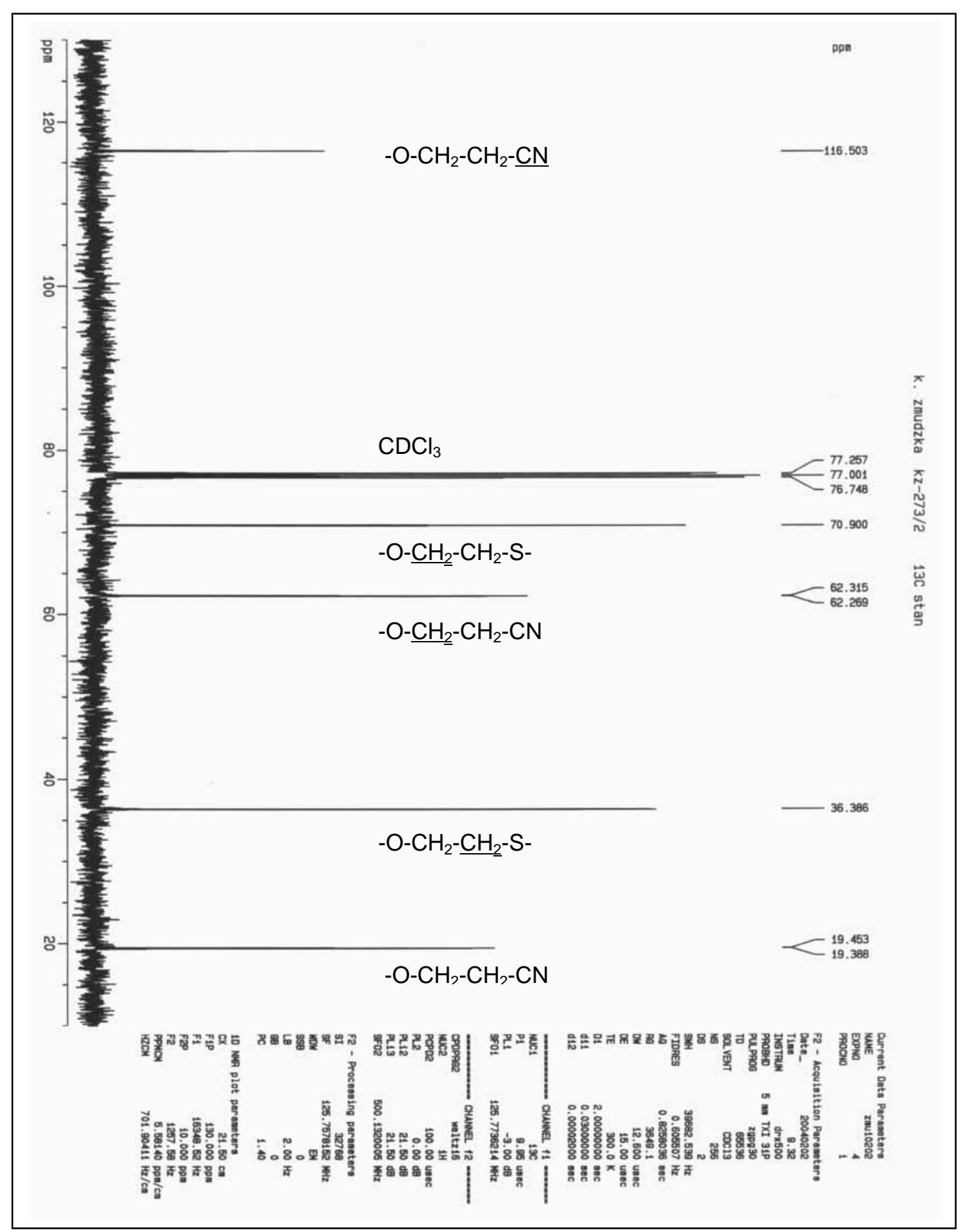


Compound 2: ${ }^{13} \mathrm{C}$ NMR spectrum with ${ }^{1} \mathrm{H}$ and ${ }^{31} \mathrm{P}$ decoupling

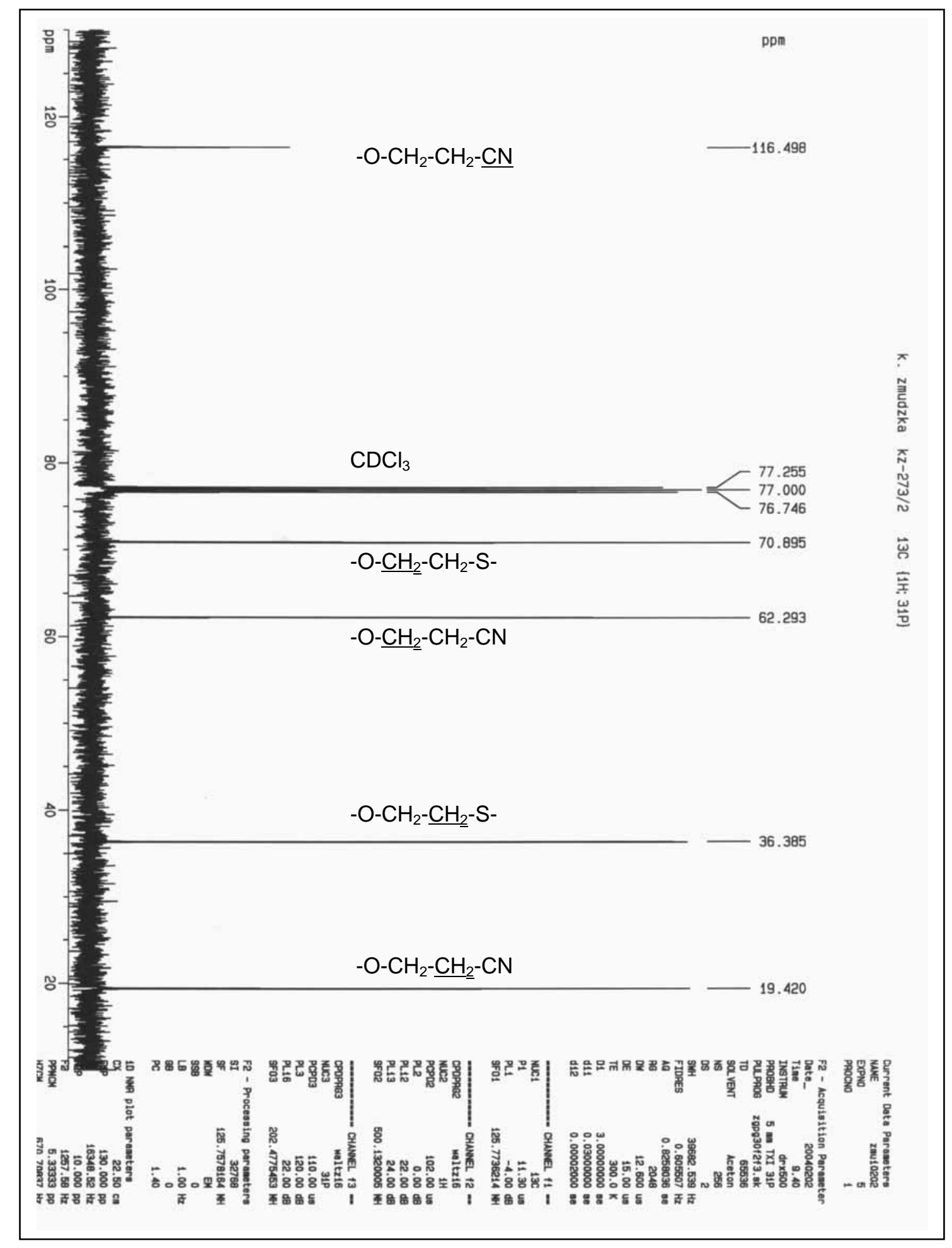


Compound 2: ${ }^{31} \mathrm{P}$ NMR spectrum

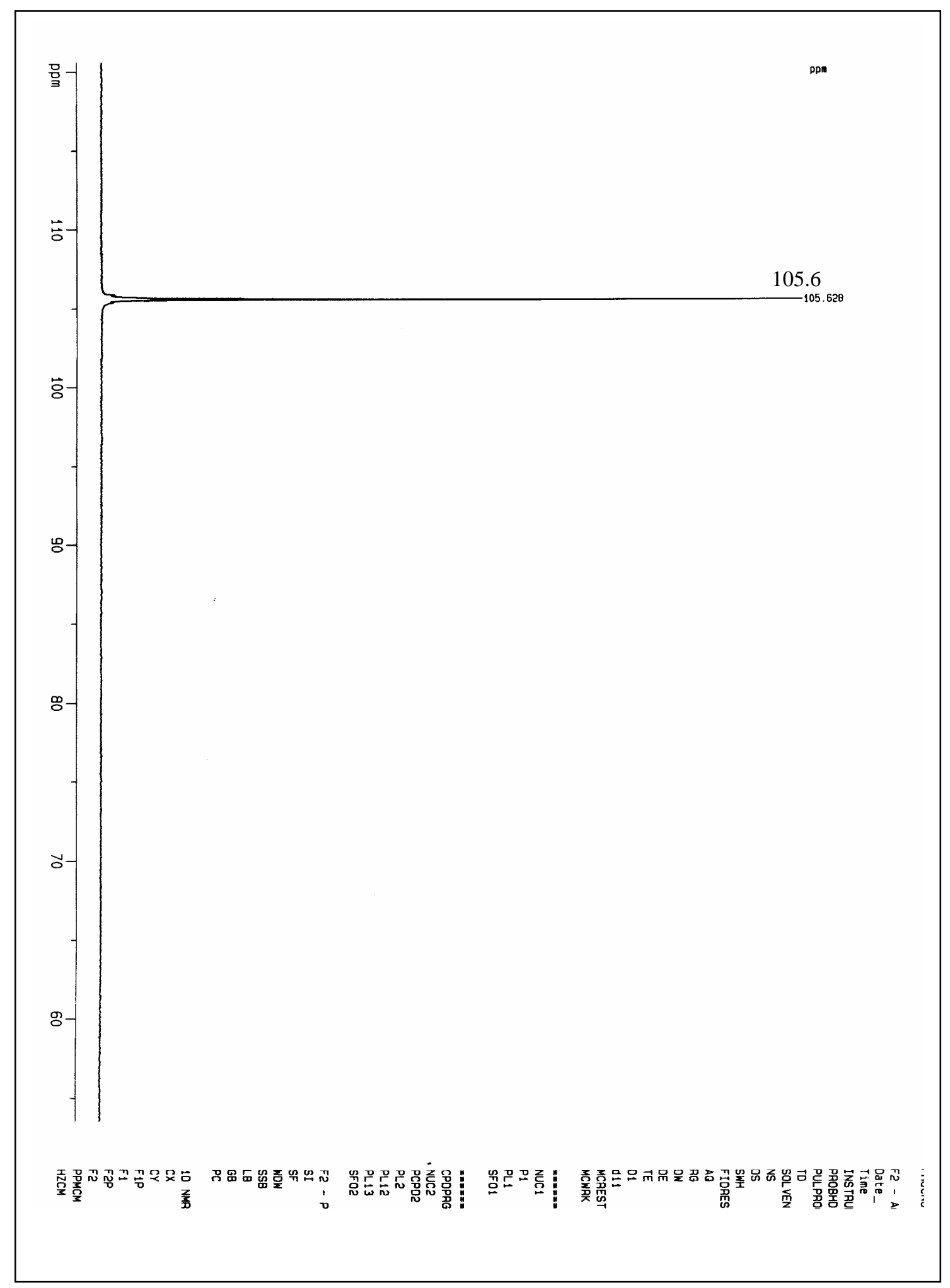


Spectral data for compounds 5 :

\section{Data for 5a:}

${ }^{31} \mathrm{P}$ NMR spectrum of crude $\mathbf{5 a}$ :

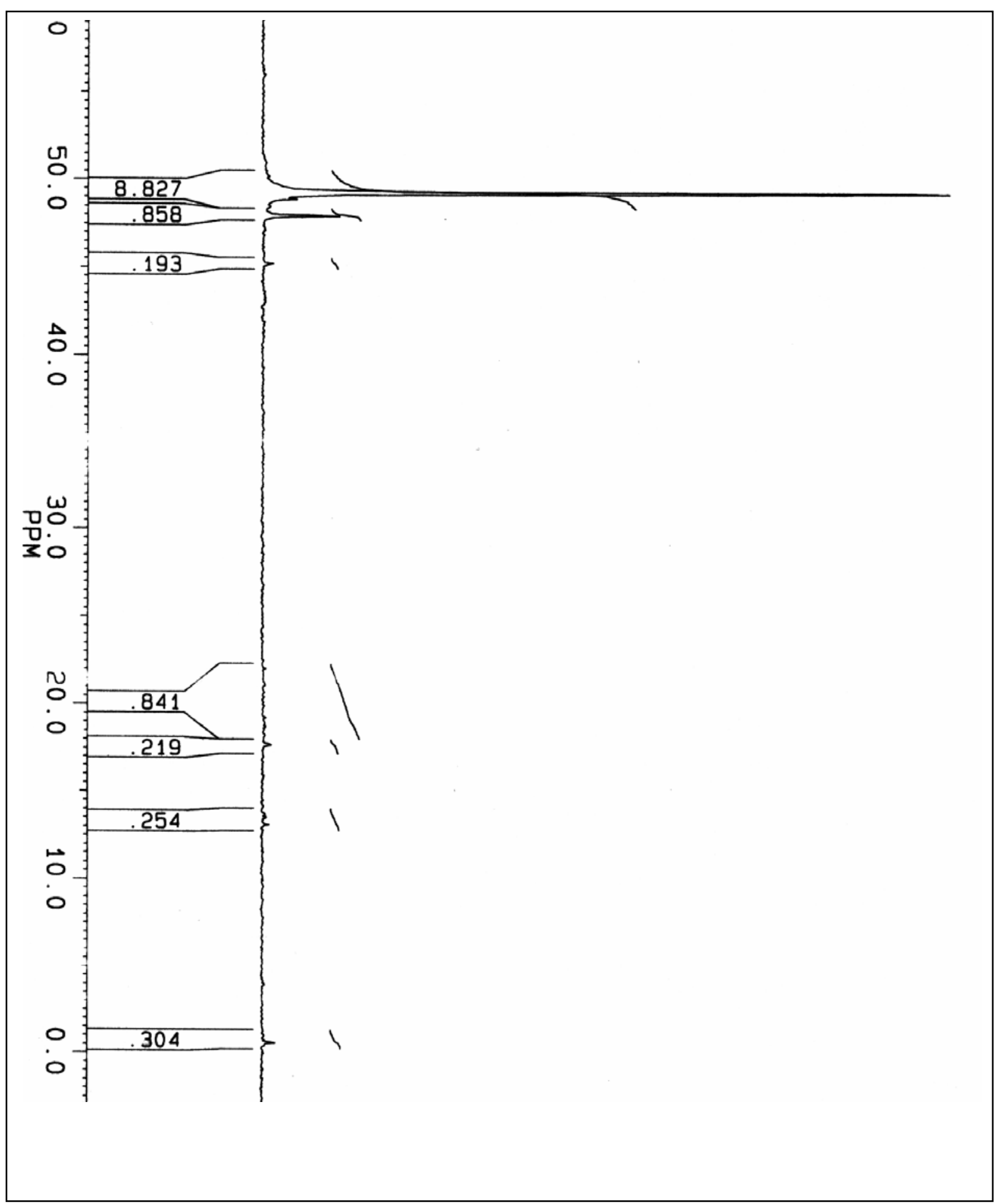


${ }^{31}$ P NMR spectrum of $\mathbf{5 a}$ after silica gel column purification:

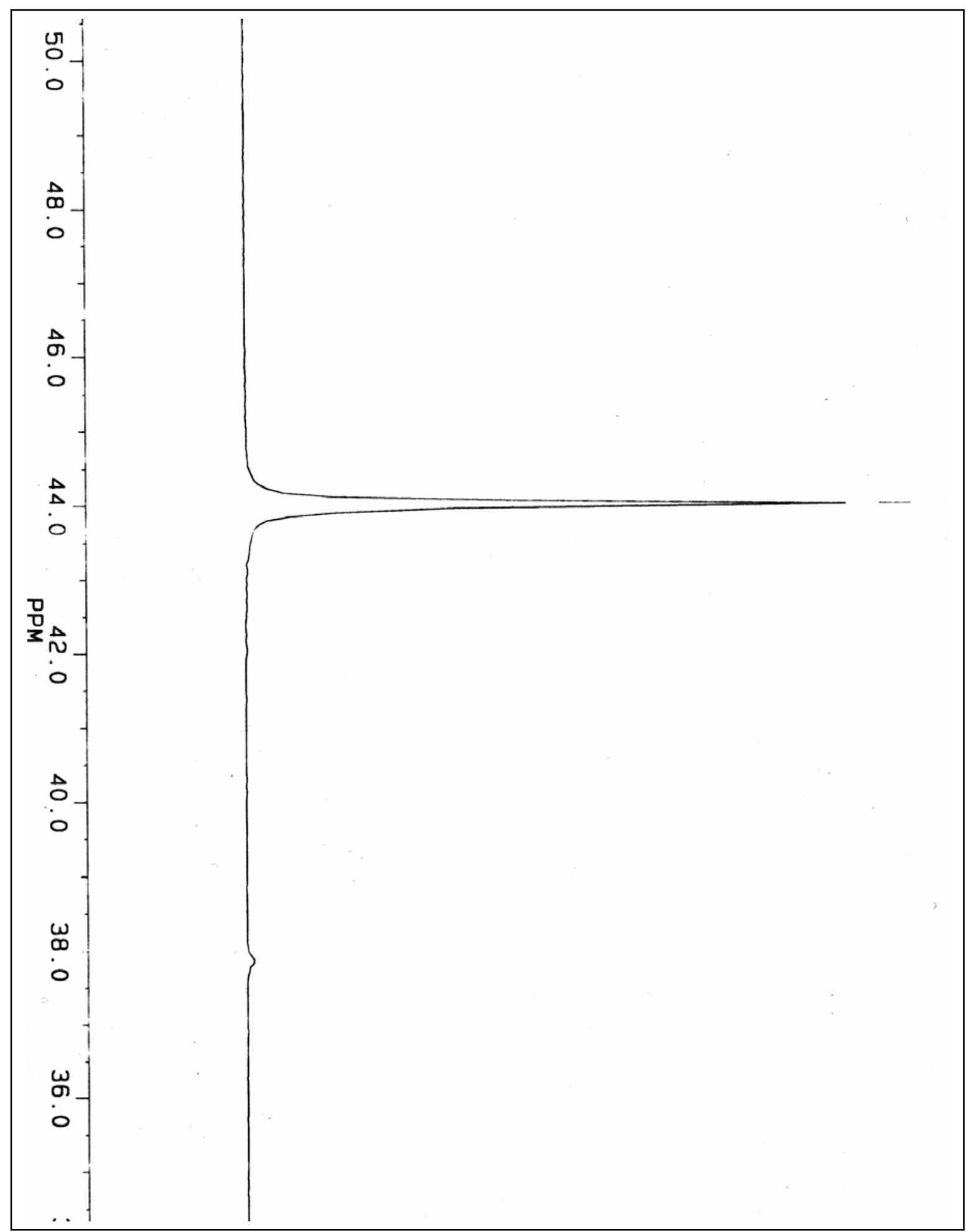


${ }^{1} \mathrm{H}$ NMR spectrum of $\mathbf{5 a}, \mathrm{DBUH}^{+} / \mathrm{NH}_{4}{ }^{+}$salt

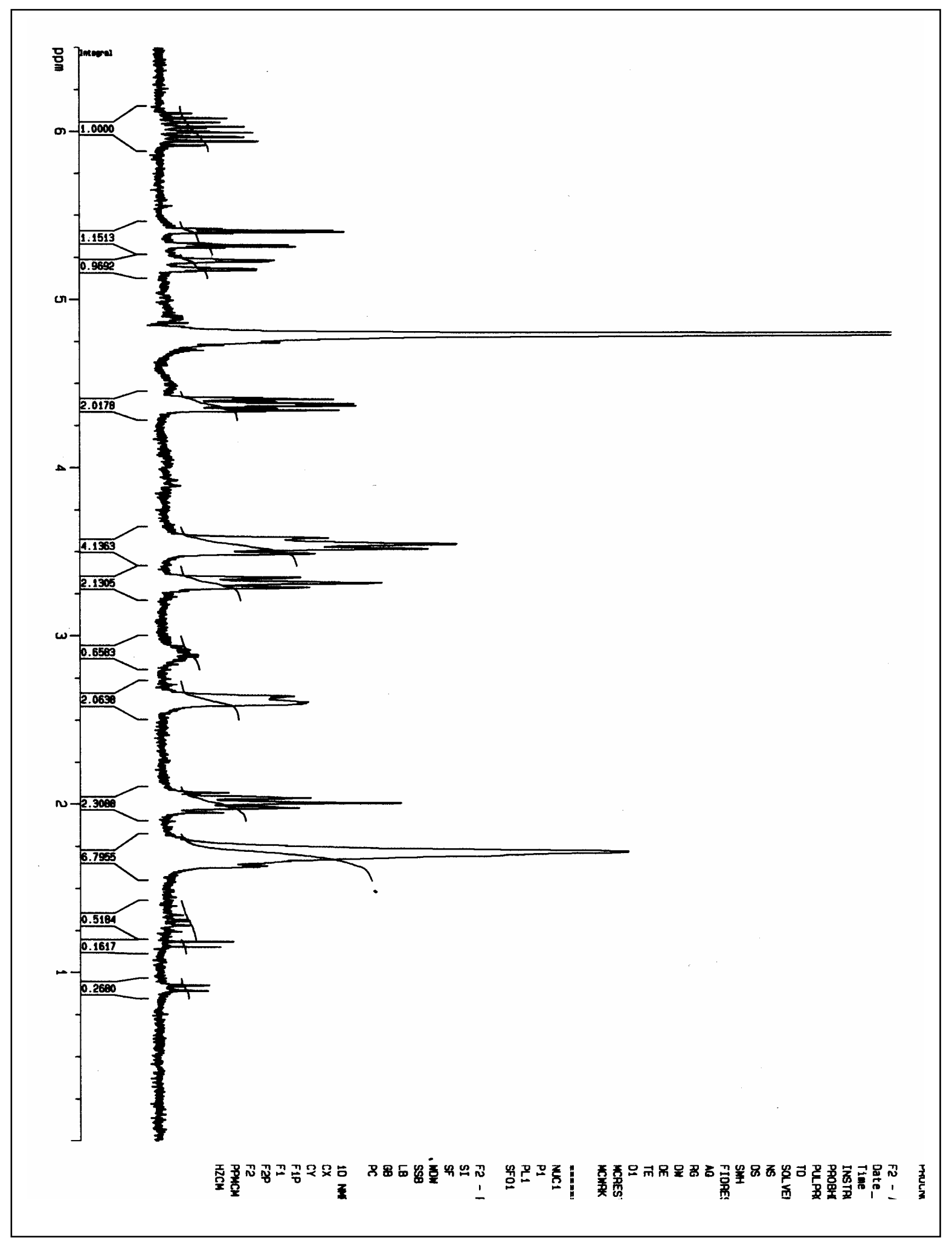


${ }^{1} \mathrm{H}$ NMR spectrum of allyl alcohol

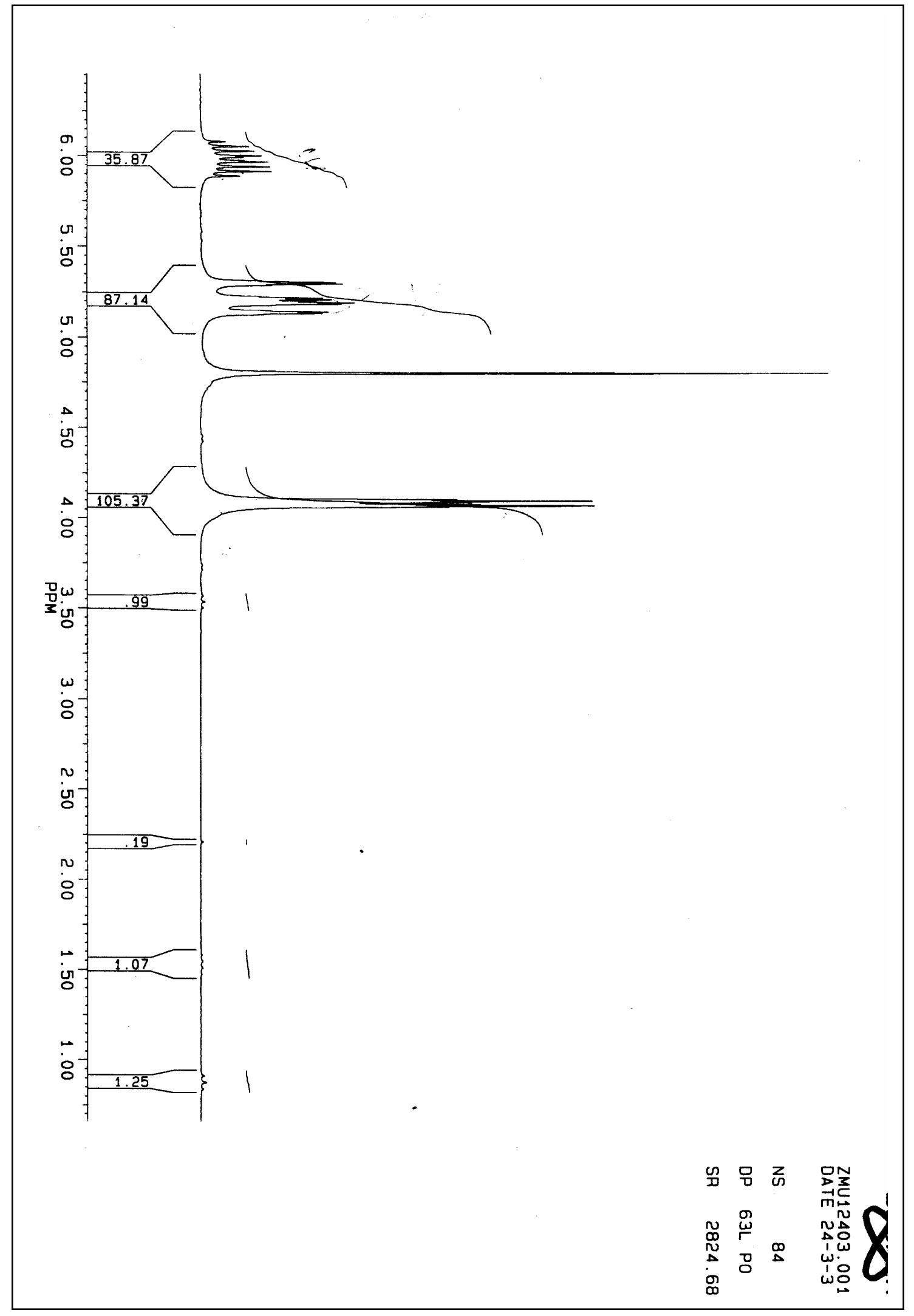


HR MS of $\mathbf{5 a}$

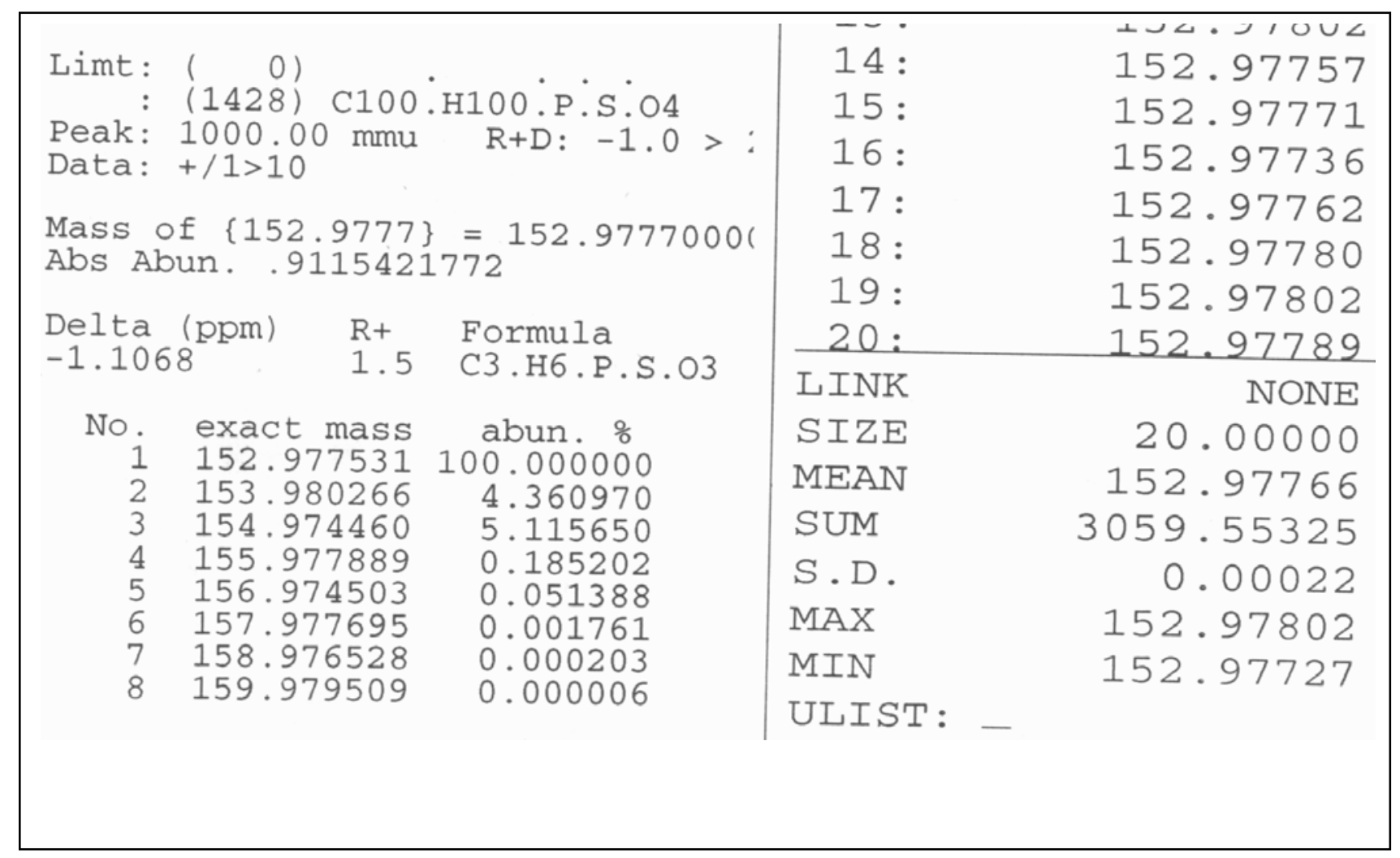


Data for $\mathbf{5 b}$ :

${ }^{31} \mathrm{P}$ NMR spectrum of $\mathbf{5 b}$ :

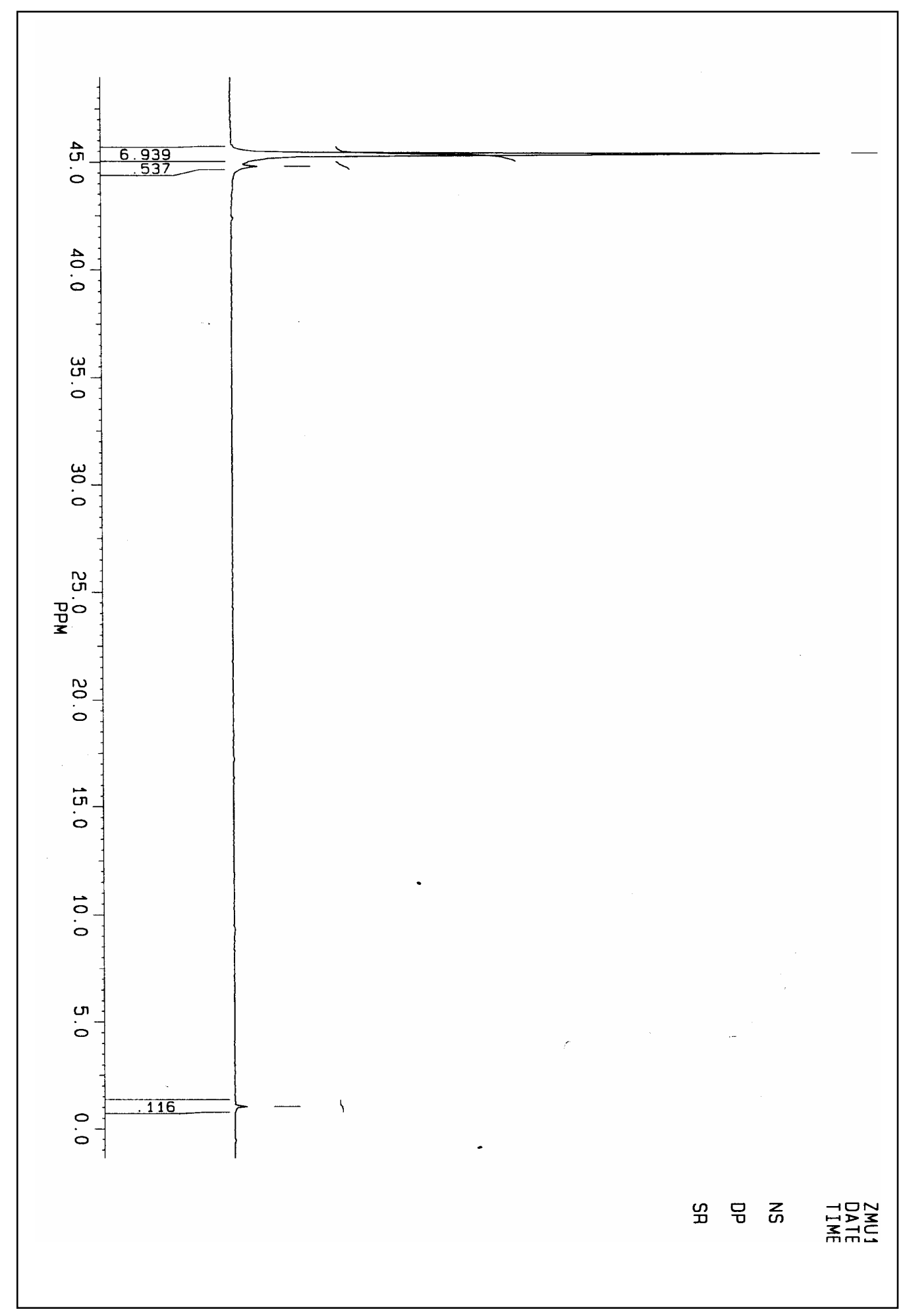


HR MS of $\mathbf{5 b}$ :

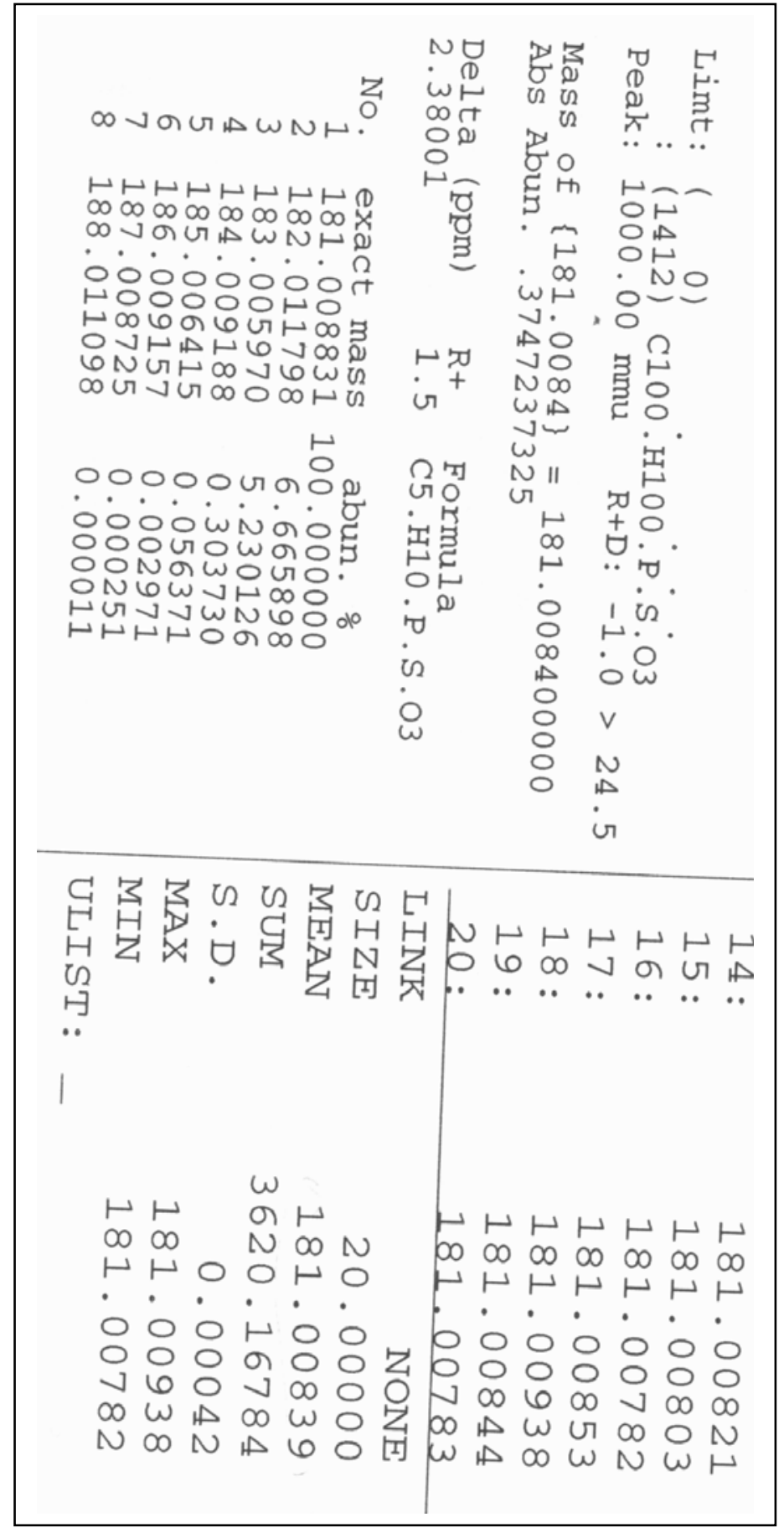


${ }^{1} \mathrm{H}$ NMR spectrum of $\mathbf{5 b}, \mathrm{DBUH}^{+} / \mathrm{NH}_{4}{ }^{+}$salt:

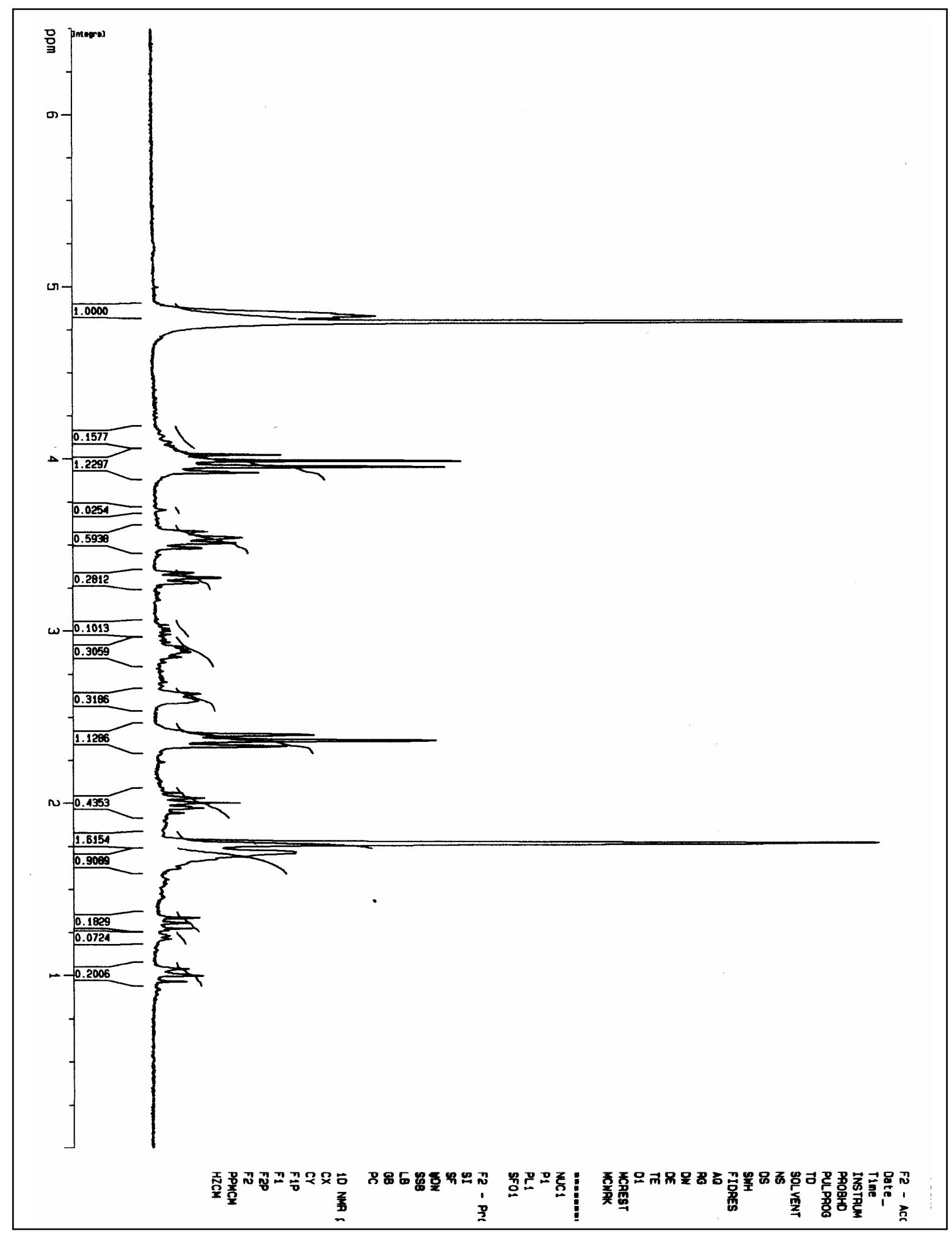


${ }^{1} \mathrm{H}$ NMR spectrum of isopentenyl alcohol:

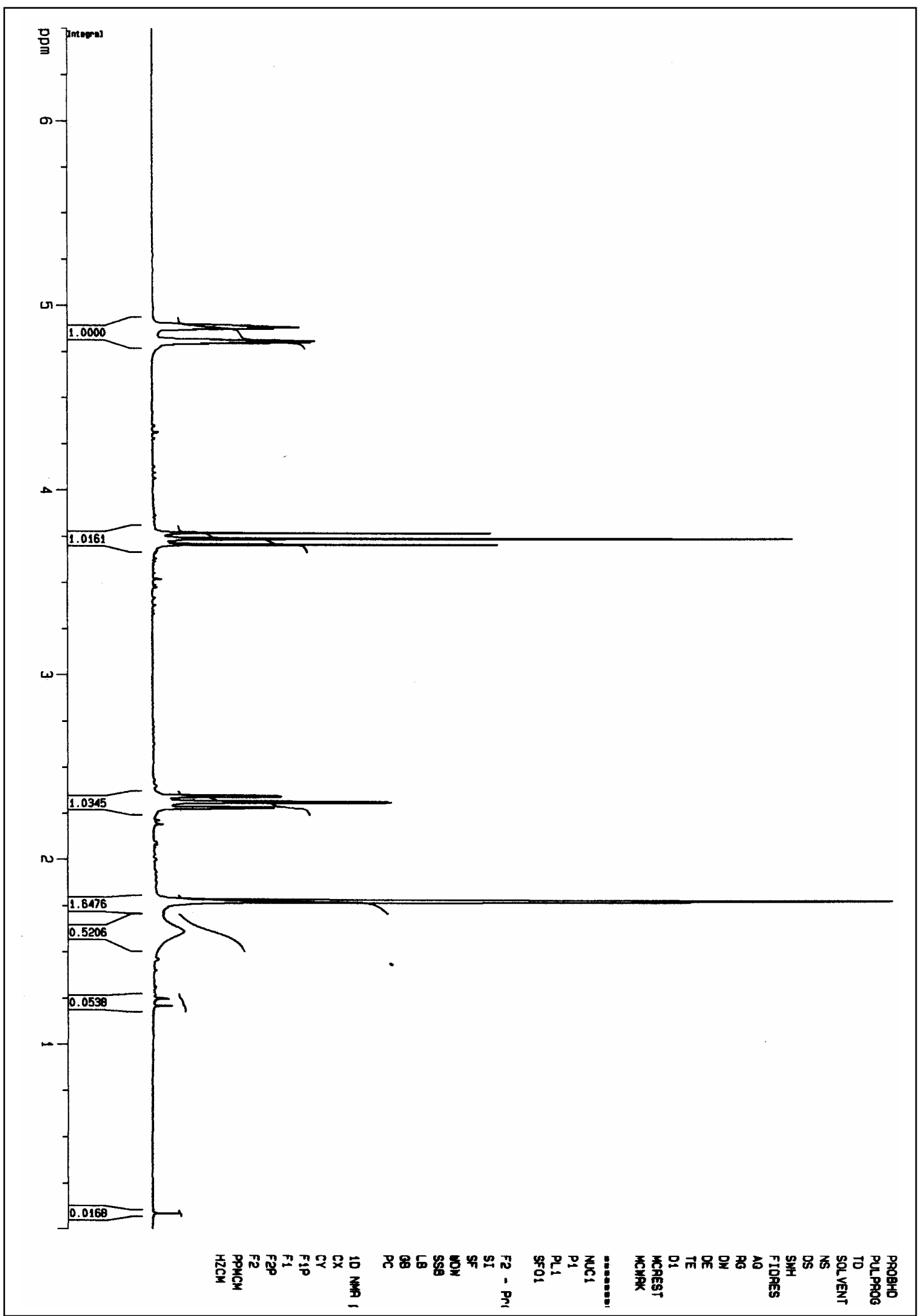


Data for 5c:

${ }^{31}$ P NMR spectrum of $\mathbf{5 c}$

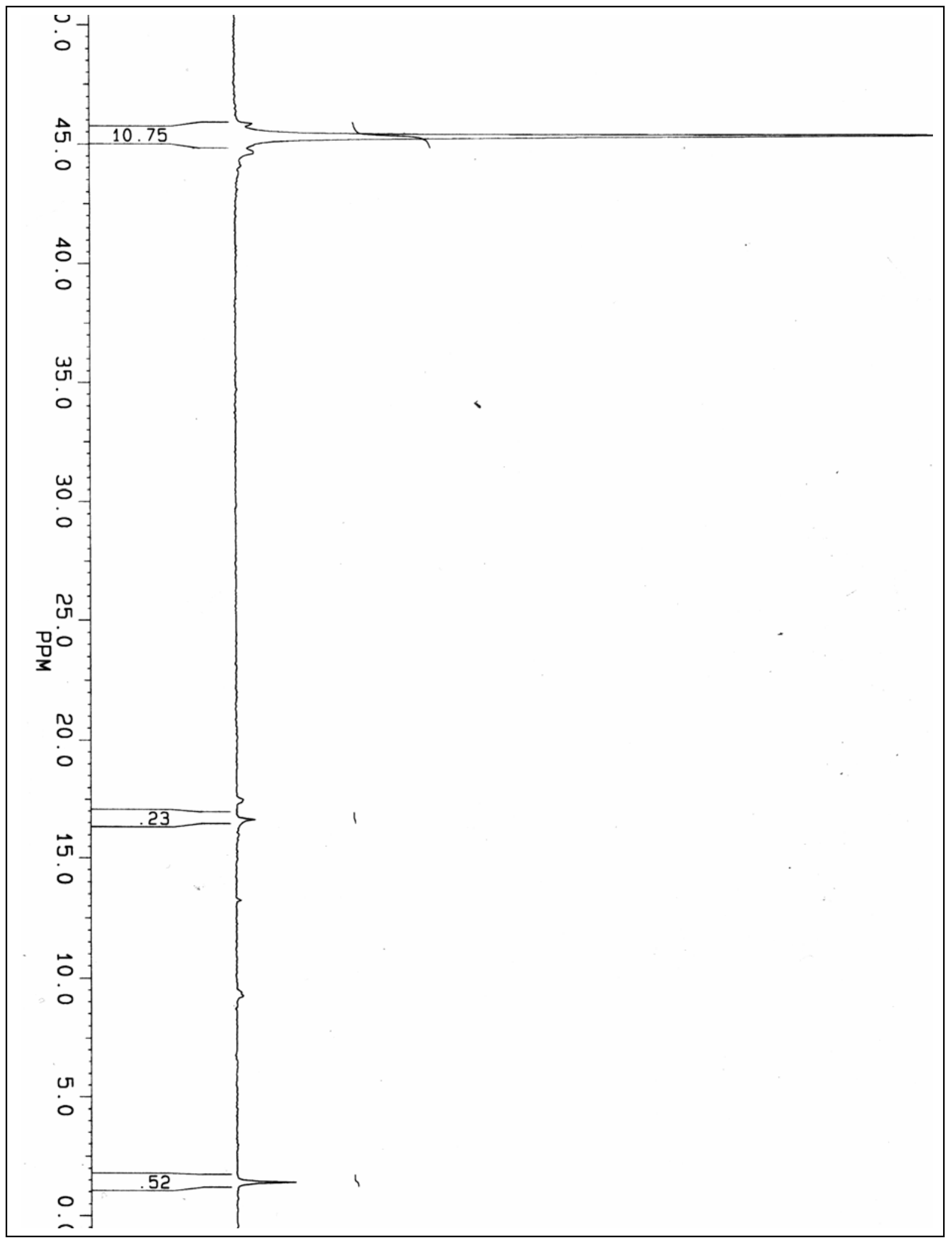


HR MS of 5c:

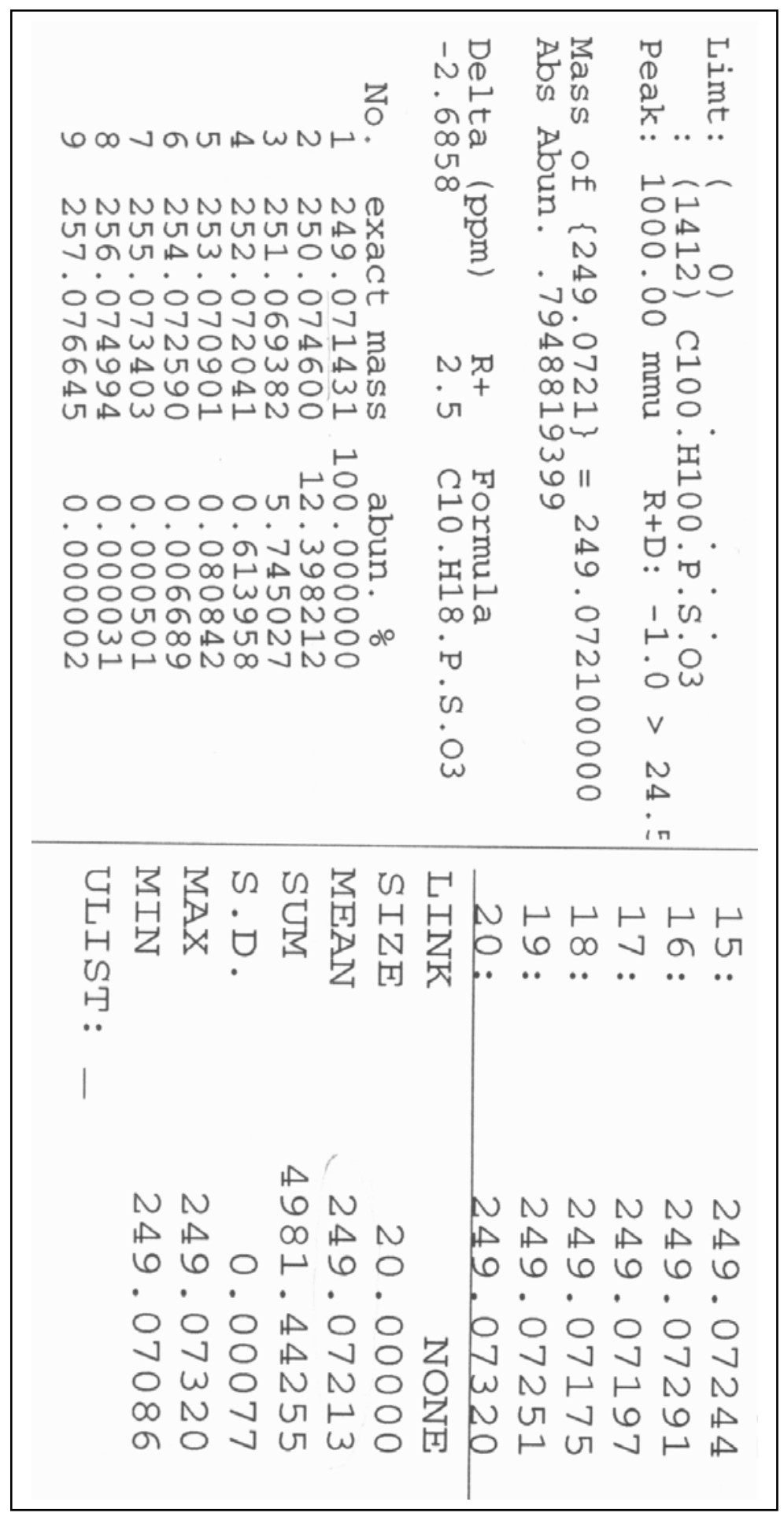


${ }^{1} \mathrm{H}$ NMR spectrum of $\mathbf{5 c}, \mathrm{DBUH}^{+} / \mathrm{NH}_{4}{ }^{+}$salt:

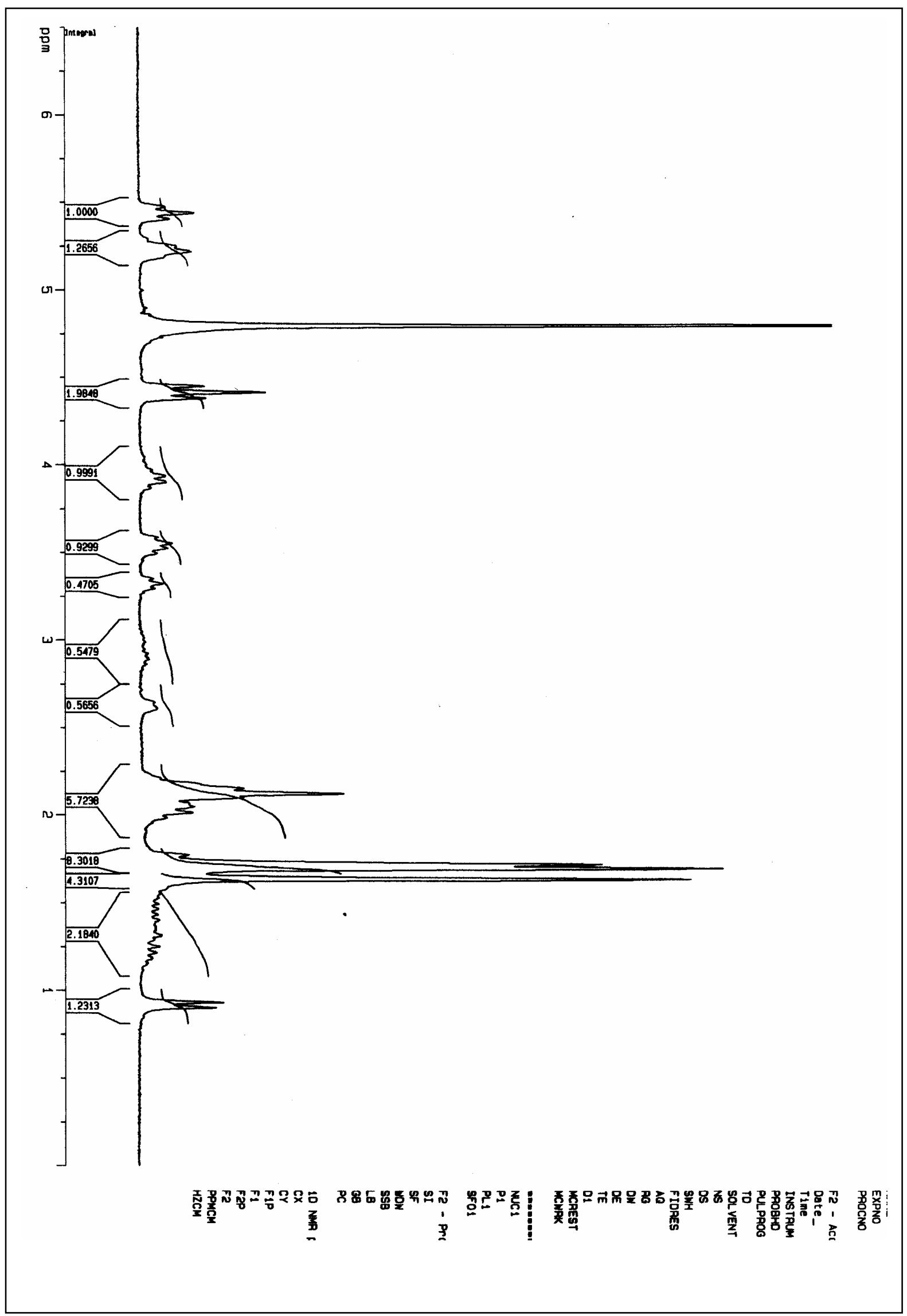


${ }^{1} \mathrm{H}$ NMR spectrum of geranyl alcohol:

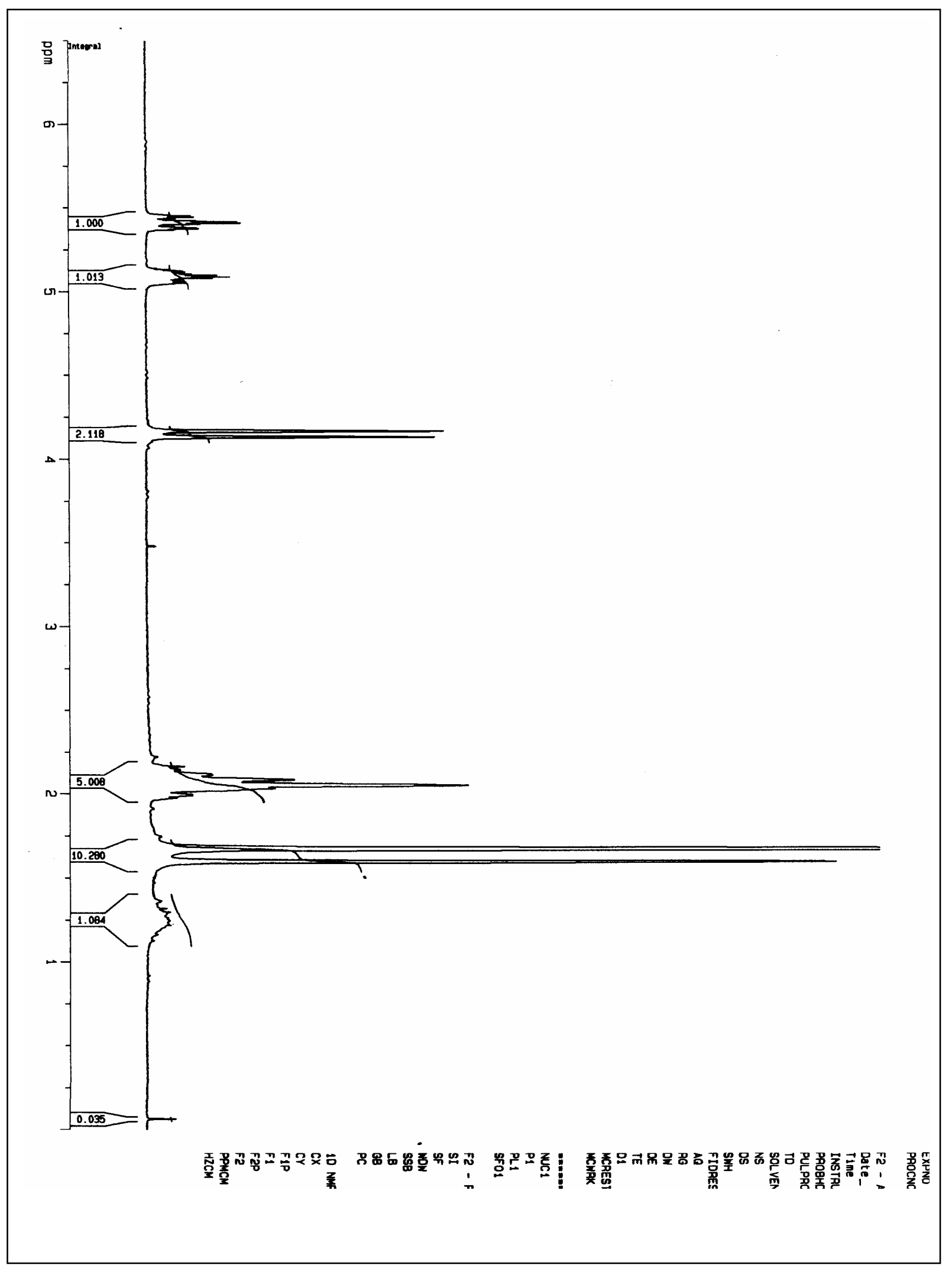


Data for 5d:

${ }^{31}$ P NMR spectrum of $\mathbf{5 d :}$

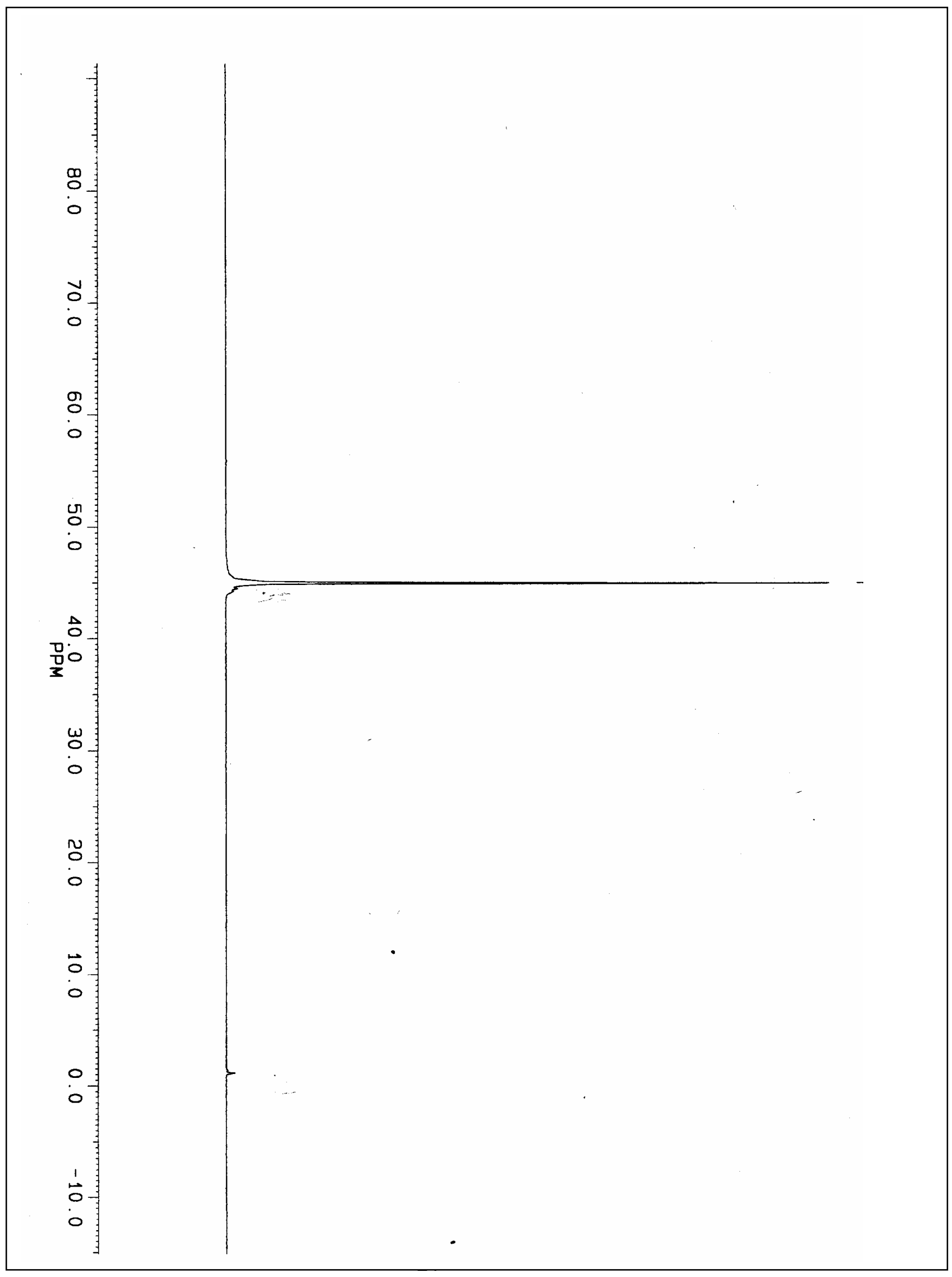


HR MS of $\mathbf{5 d :}$

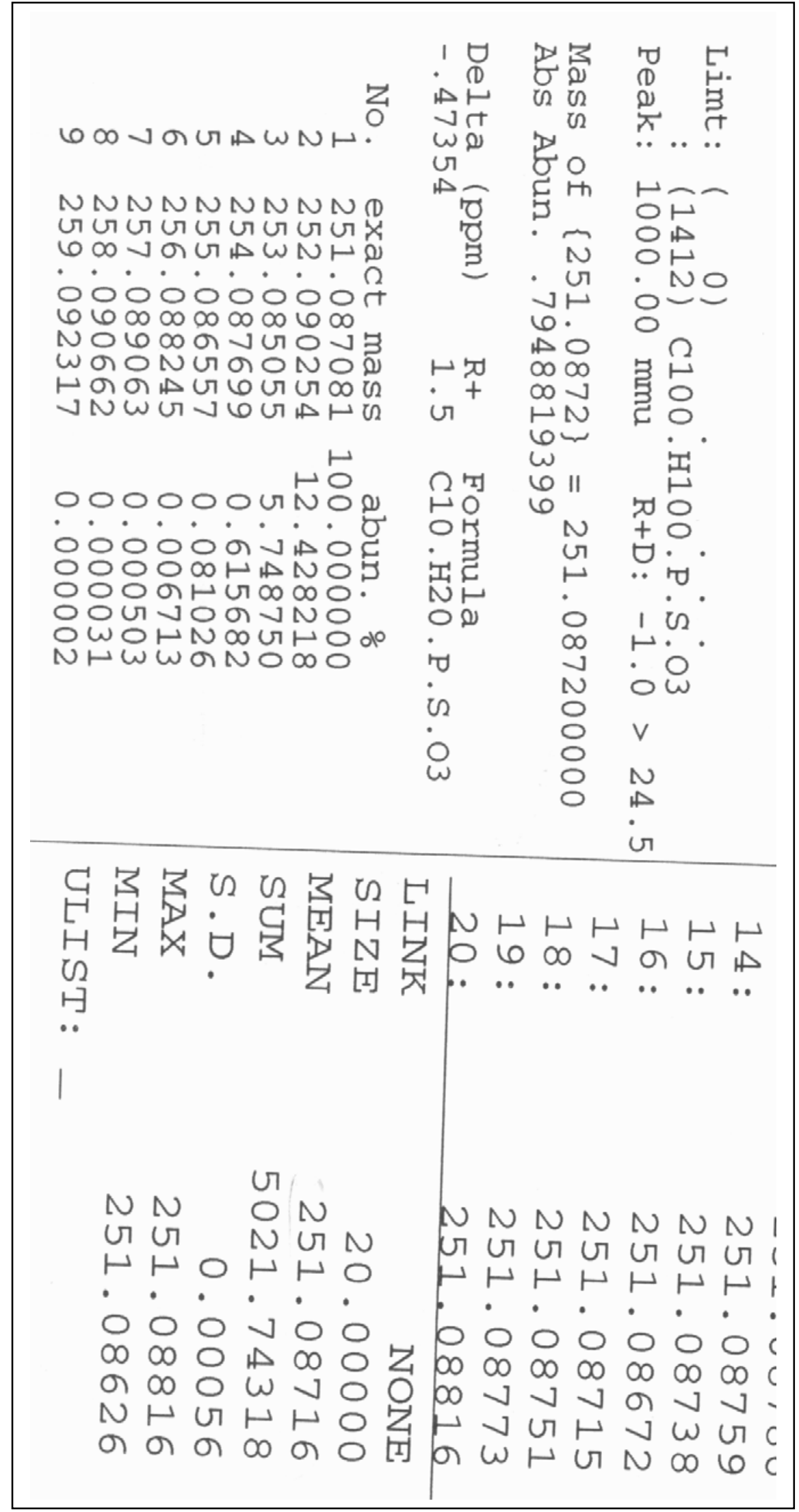


${ }^{1} \mathrm{H}$ NMR spectrum of $\mathbf{5 d}$, $\mathrm{DBUH}^{+} / \mathrm{NH}_{4}{ }^{+}$salt:

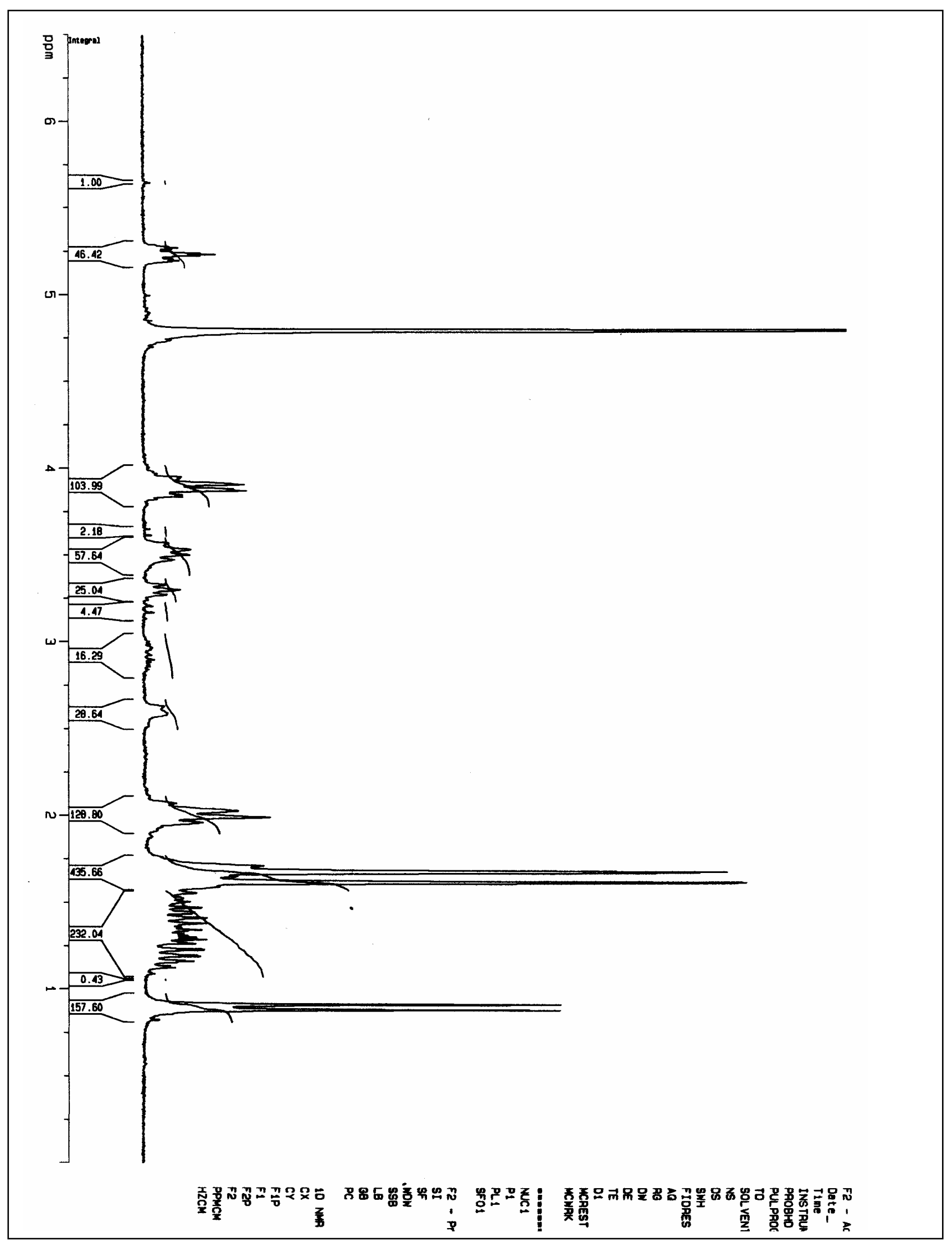


${ }^{1} \mathrm{H}$ NMR spectrum of citronellyl alcohol:

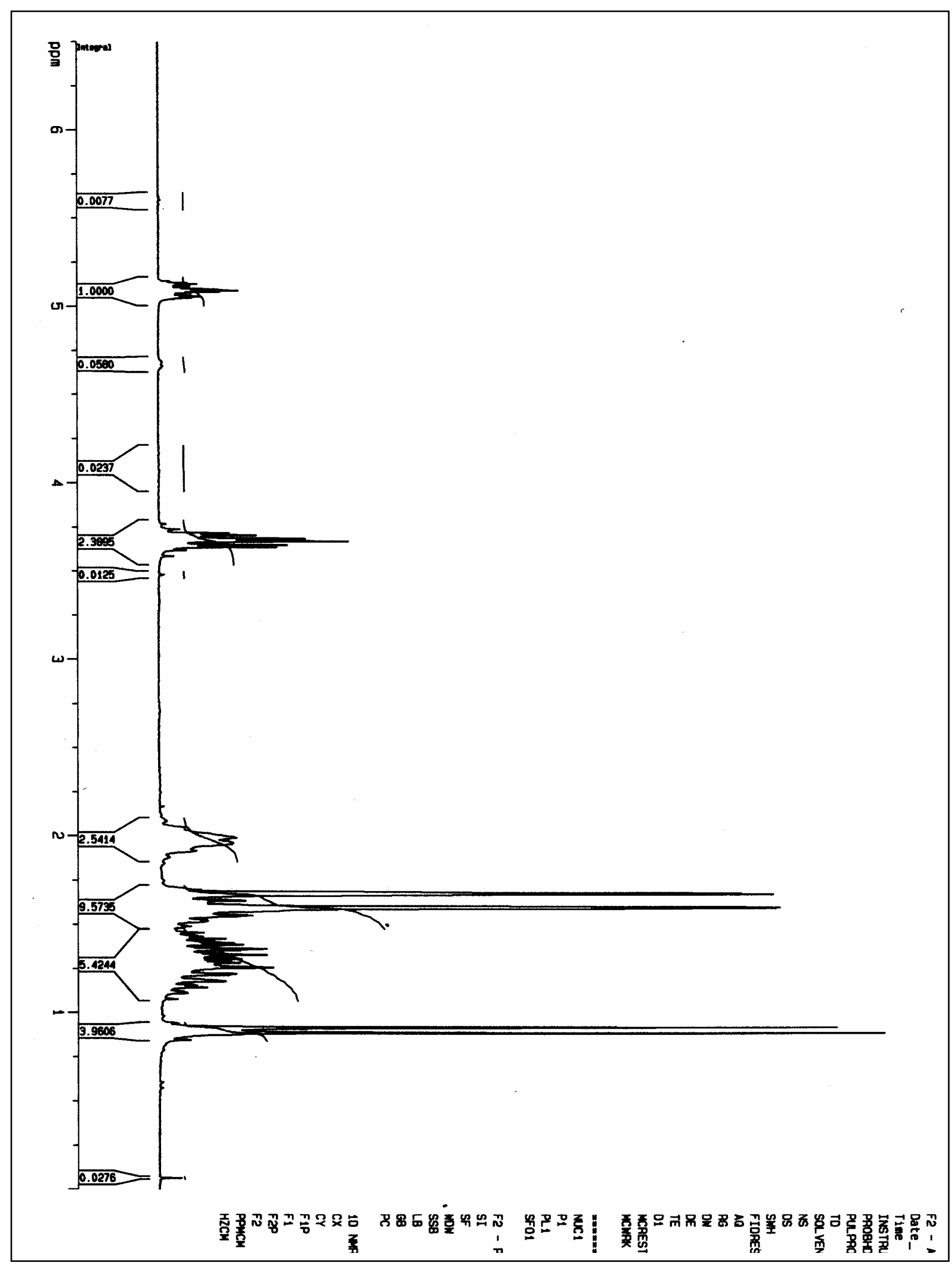


Data for 5e:

${ }^{31}$ P NMR spectrum of $\mathbf{5 e}$ :

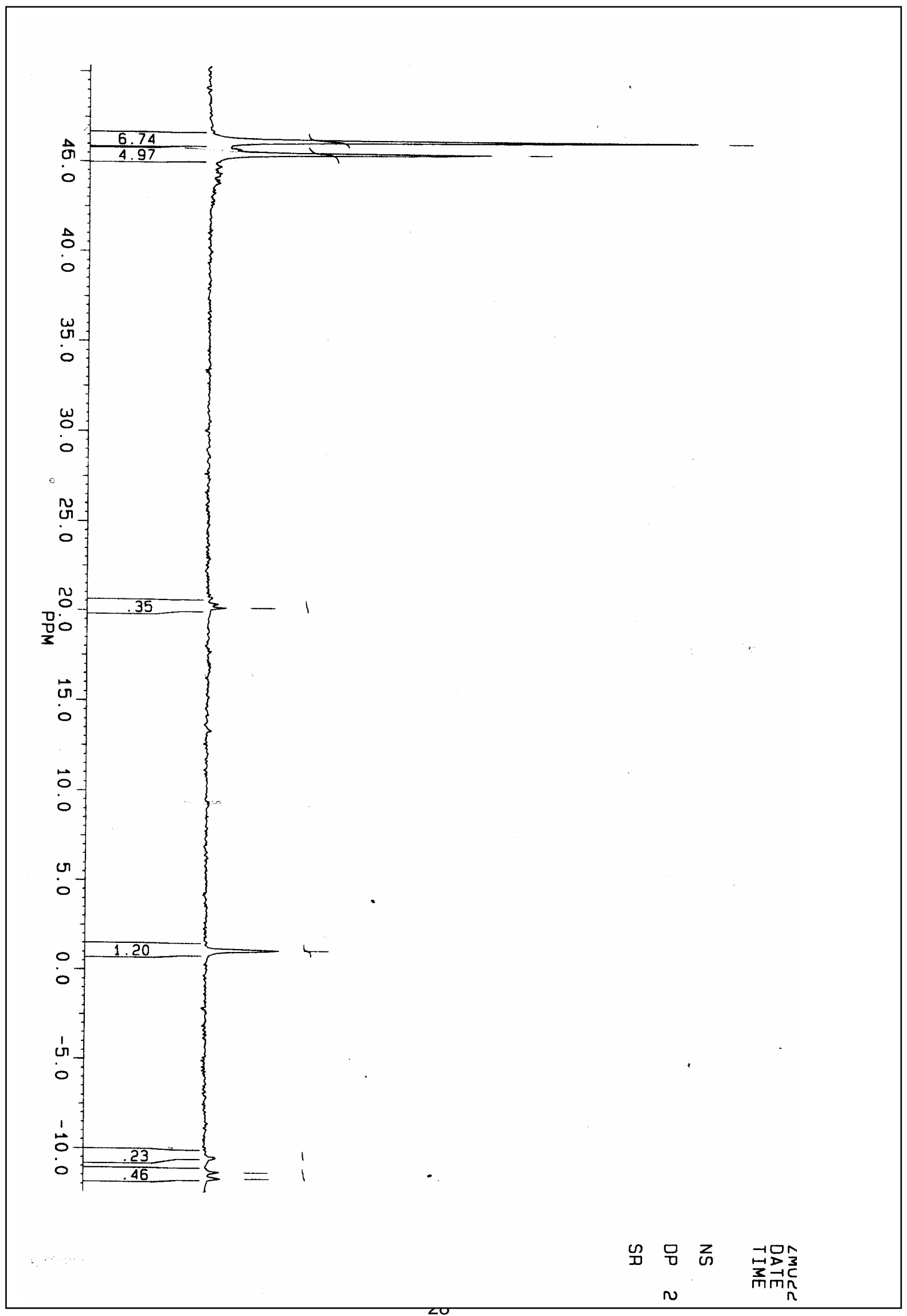


HR MS of 5e:

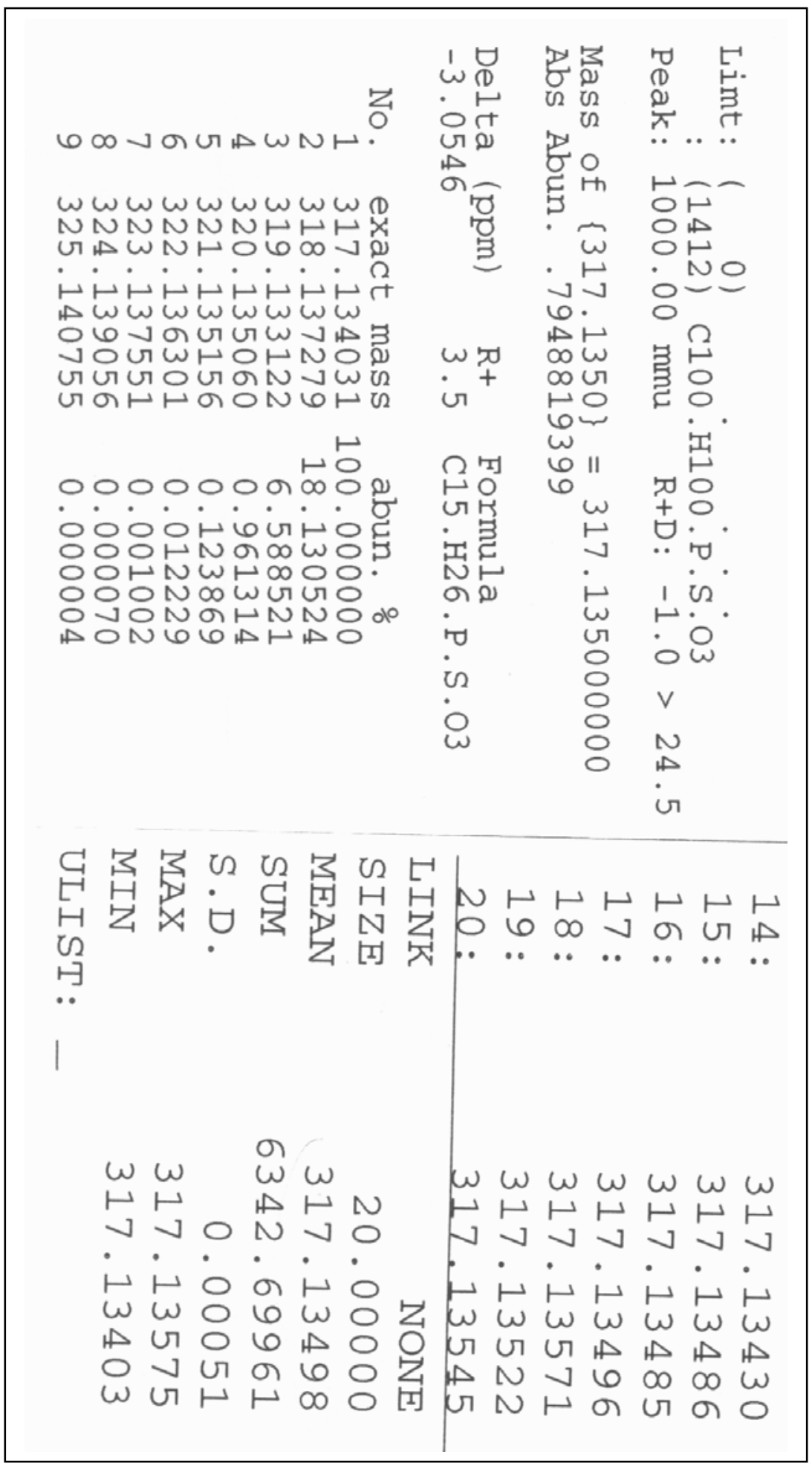


${ }^{1} \mathrm{H}$ NMR spectrum of $\mathbf{5 e}, \mathrm{NH}_{4}{ }^{+}$salt:

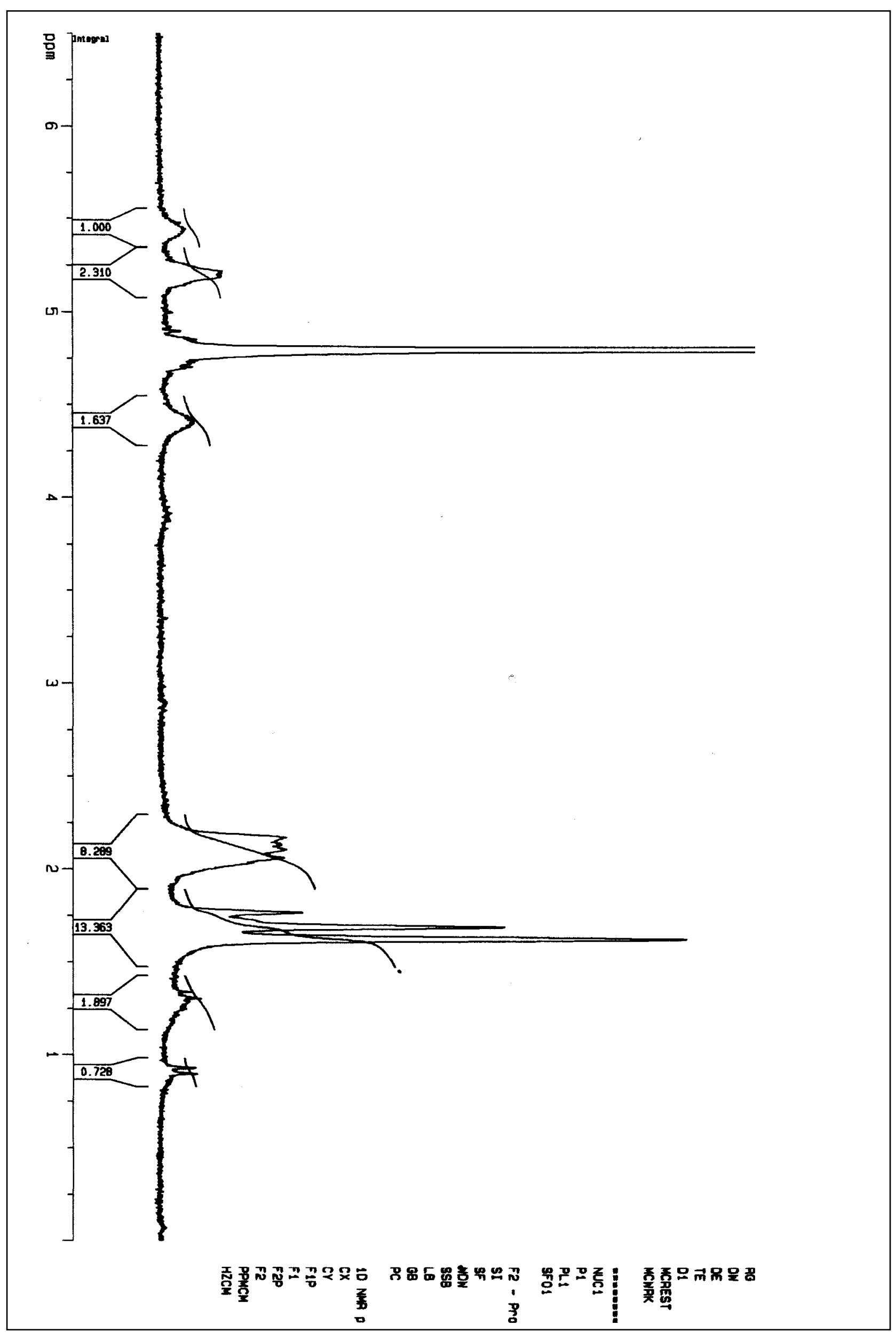


${ }^{1}$ H NMR spectrum of farnesyl alcohol:

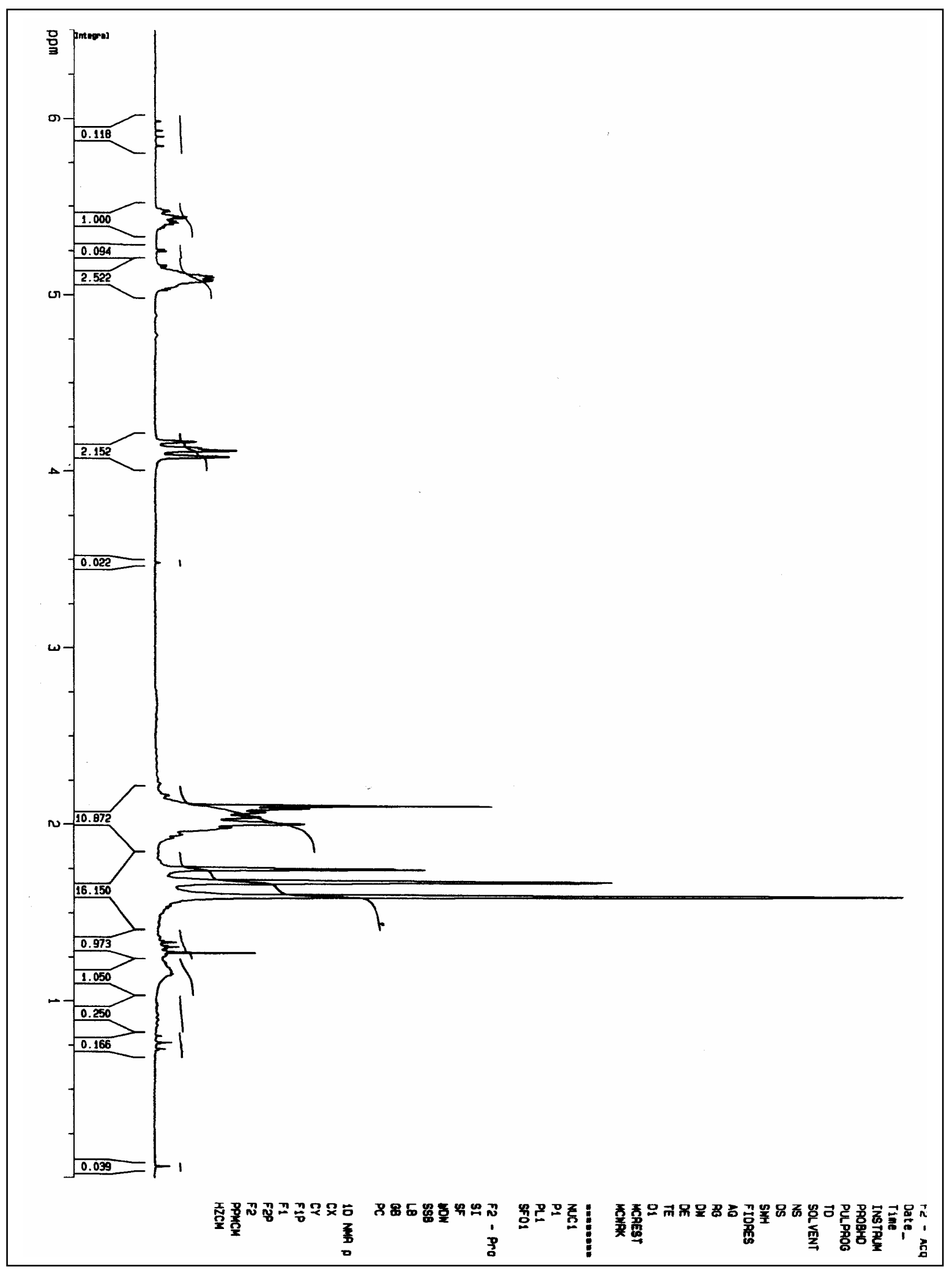


Data for $\mathbf{5 f}$ :

${ }^{31}$ P NMR spectrum of $\mathbf{5 f}$ :

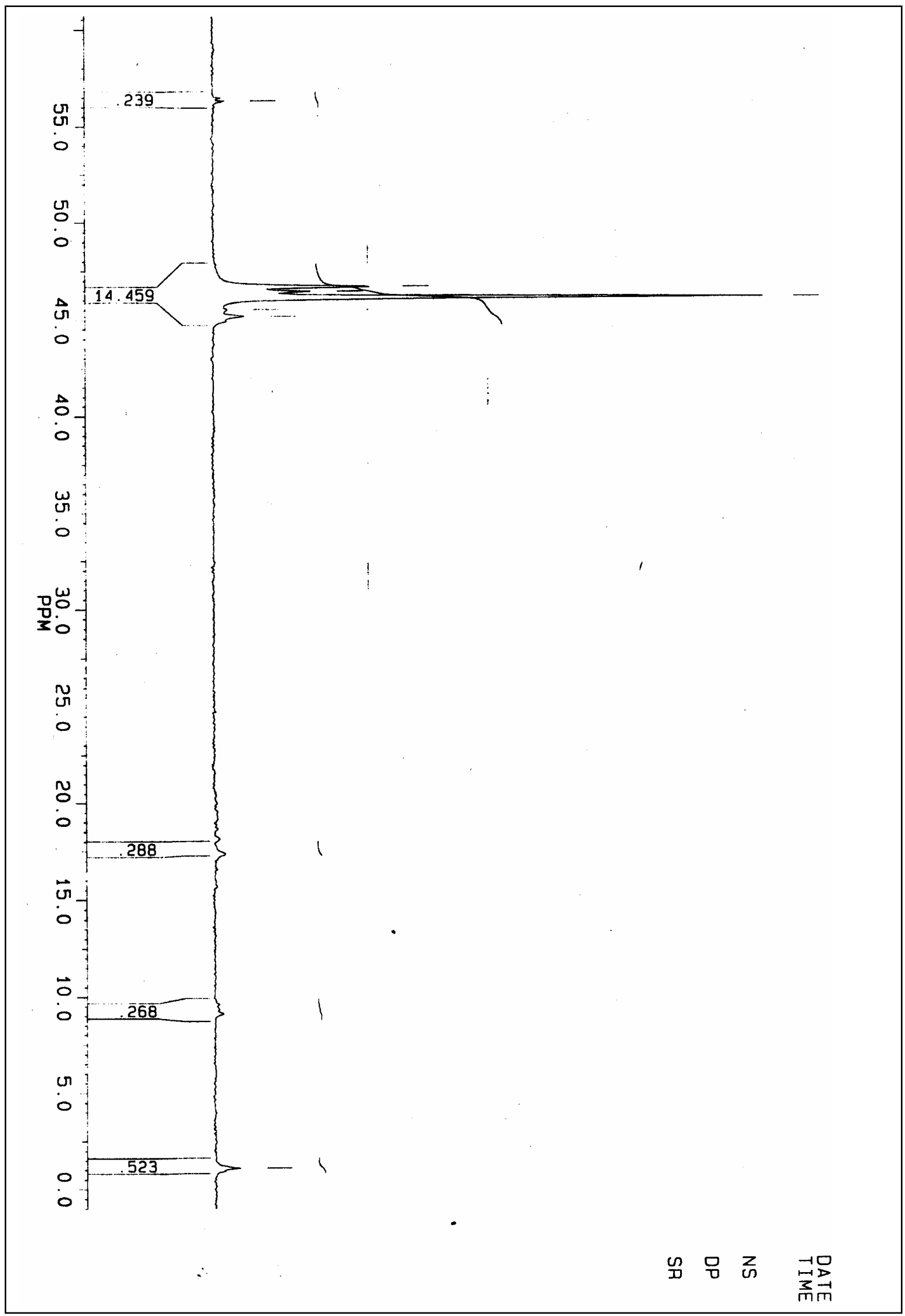


HR MS of $\mathbf{5 f}$ :

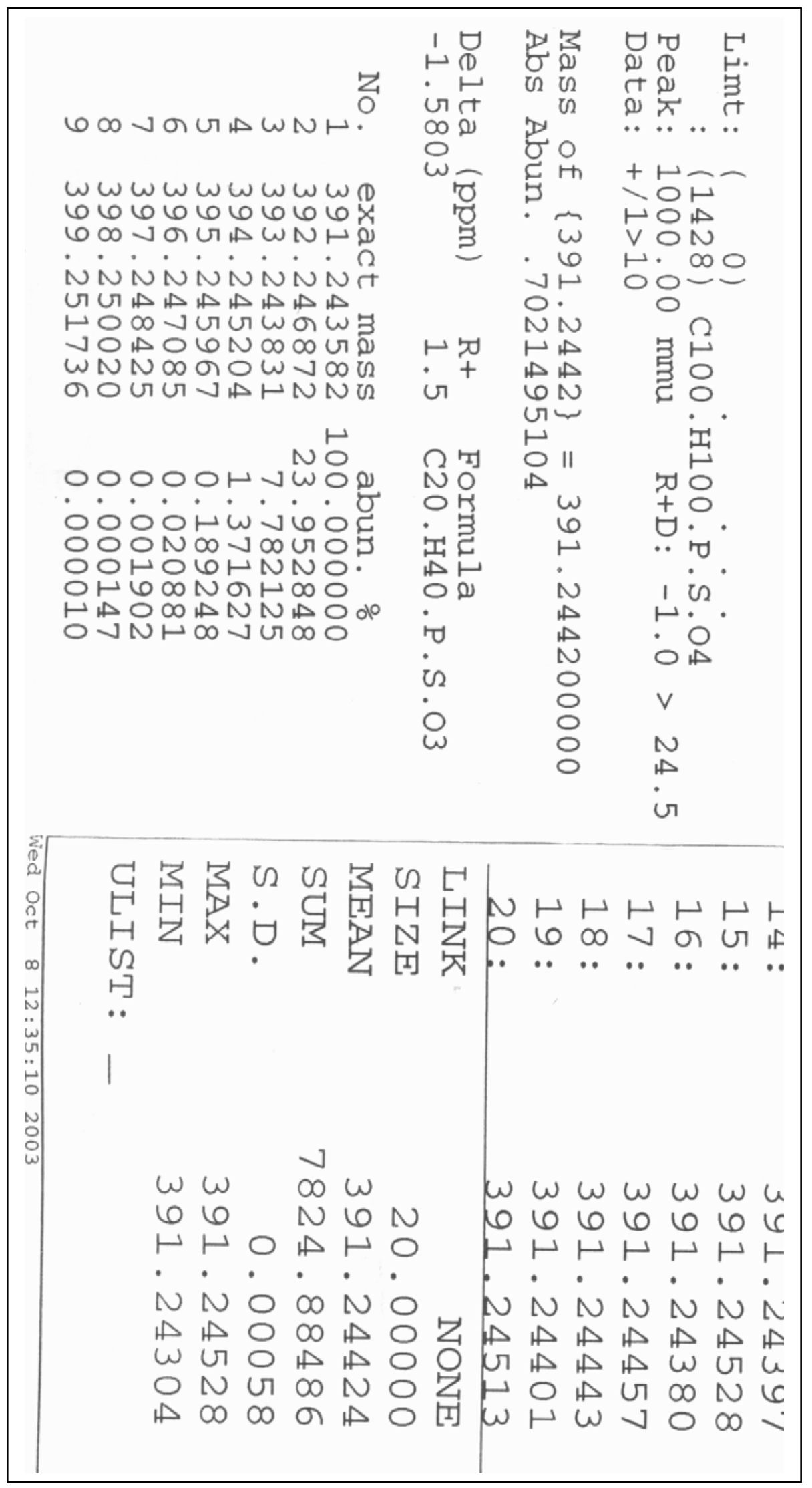


${ }^{1} \mathrm{H}$ NMR spectrum of $\mathbf{5 f}, \mathrm{DBUH}^{+} / \mathrm{NH}_{4}{ }^{+}$salt:

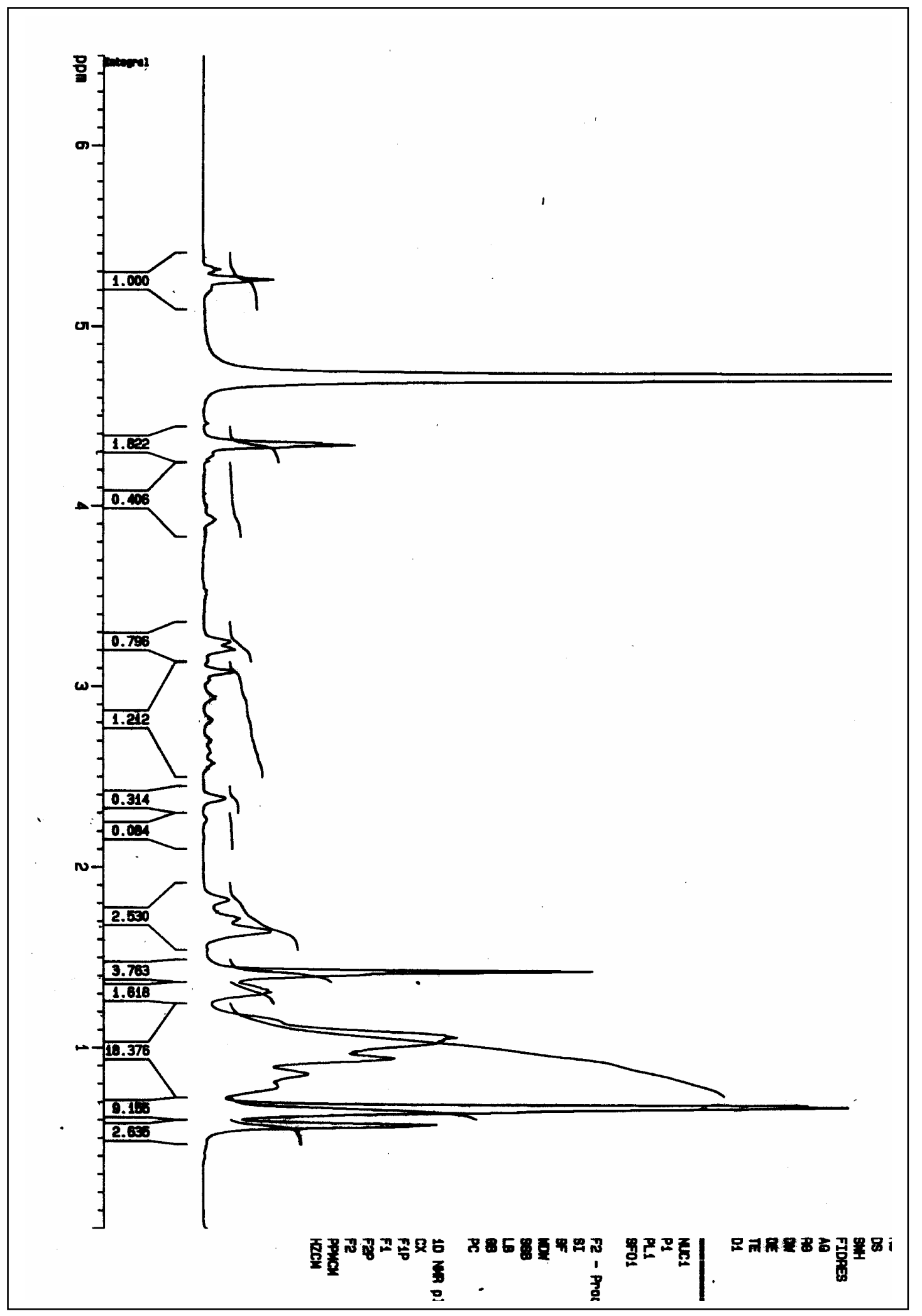


${ }^{1}$ H NMR spectrum of phytyl alcohol:

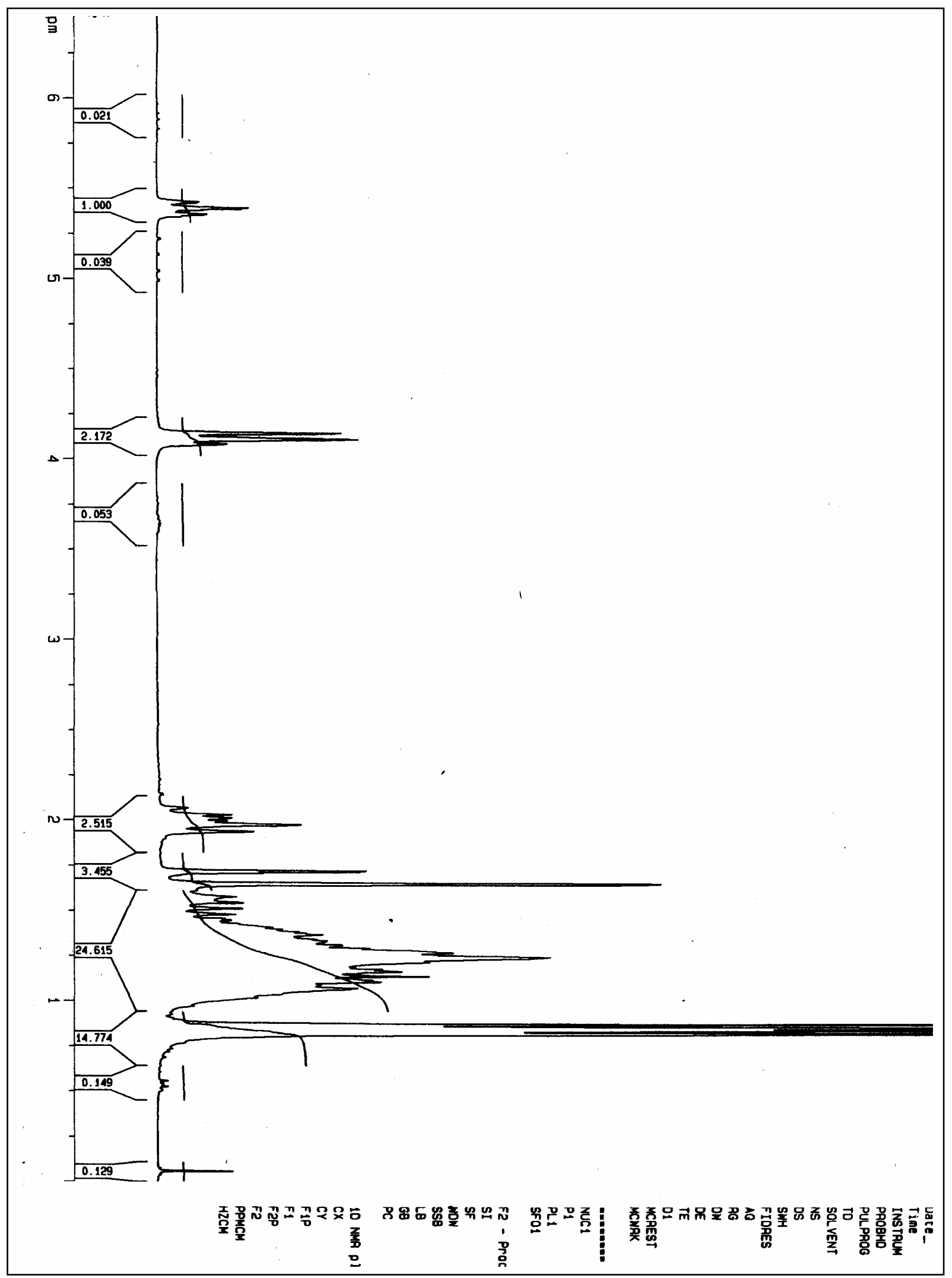

